\title{
On several new or poorly-known Oriental Paradoxosomatidae (Diplopoda: Polydesmida), XIX
}

\section{О несколыких новых или плохоизученных ориенталыных Paradoxosomatidae (Diplopoda: Polydesmida), XIX}

\author{
S.I. Golovatch \\ С.И. Головач
}

Institute for Problems of Ecology and Evolution, Russian Academy of Sciences, Leninsky prospekt 33, Moscow 119071 Russia. E-mail: sgolovatch@yandex.ru

Институт проблем экологии и эволюции РАН, Ленинский проспект, 33, Москва 119071 Россия.

KEY WORDS: Diplopoda, Polydesmida, Paradoxosomatidae, taxonomy, new records, new species, Nepal, China, Myanmar, Vietnam, Laos, Japan.

КЛЮЧЕВЫЕ СЛОВА: Diplopoda, Polydesmida, Paradoxosomatidae, таксономия, новые находки, новые виды, ключ, Непал, Китай, Мьянма, Вьетнам, Лаос, Япония.

ABSTRACT. This contribution is devoted to new records of several known, and to descriptions of the following eight new, species: Chamberlinius vietnamicus sp.n. and Sundanina fedorenkoi sp.n., both from Vietnam, Delarthrum quadridentatum sp.n. and Touranella pilosa sp.n., both from Nepal, Pocockina jaegeri sp.n. and Tetracentrosternus martensi sp.n., both from Myanmar, and Inversispina multispina sp.n. and Sigipinius pinnifer sp.n., both from Sichuan, China. Keys are given to all six, three or four presently known species of Tou ranella, Pocockina and Tetracentrosternus, respectively.

РЕЗЮМЕ. Данное сообщение посвящено находкам нескольких известных, а также описаниям следующих восьми новых видов: Chamberlinius vietnamicus sp.n. и Sundanina fedorenkoi sp.n., оба из Вьетнама, Delarthrum quadridentatum sp.n. и Touranella pilosa sp.n., оба из Непала, Pocockina jaegeri sp.n. и Tetracentrosternus martensi sp.n., оба из Мьянмы, и Inversispina multispina sp.n. и Sigipinius pinnifer sp.n., оба из Сычуани (Китай). Даны ключи для соответственно всех ныне известных шести, трех и четырех видов родов Touranella, Pocockina и Tetracentrosternus.

\section{Introduction}

This paper is devoted to new records of a few known, as well as to descriptions of eight new, species of paradoxosomatid millipedes from Nepal, Myanmar, China, Vietnam, Laos and Japan.

\section{Material and methods}

Most of the material is deposited in the collection of the Zoological Museum, Moscow State University, Russia (ZMUM). Several samples have been returned or donated to the Natur-Museum Senckenberg (SMF), Frankfurt a.M., Germany, the Muséum national d'Histoire naturelle, Paris (MNHN), France, and the Museo Civico di Storia Naturale, Verona (MCSNV), Italy, as indicated below.

Most of the focus stacking pictures were taken in Moscow, Russia, but a few also at the Royal Museum for Central Africa, Tervuren (MRAC), Belgium.

Taxonomic part

Antheromorpha rosea Golovatch, 2013

MATERIAL. 2 우 (ZMUM 03052 ), Laos, Vientiane Prov., Van Vieng, ca $260-290 \mathrm{~m}$ a.s.1., from $18^{\circ} 55^{\prime} 37^{\prime \prime} \mathrm{N}, 102^{\circ} 20^{\prime} 57^{\prime \prime} \mathrm{E}$ to $18^{\circ} 55^{\prime} 51^{\prime \prime} \mathrm{N}, 102^{\circ} 24^{\prime} 38^{\prime \prime} \mathrm{E}$, dry stream bed, 7-9.XI.2015, leg. I. Melnik.

REMARKS. Originally described [Golovatch, 2013a], and very soon after that reported from neartopotypes [Golovatch, 2013b], from Gaoligong Shan Mountains, Yunnan, China, this large, in vivo pink species has since been recorded in northern Thailand [Likhitrakarn et al., 2016]. Above is the first formal record of $A$. rosea in Laos.

Kronopolites biagrilectus Hoffman, 1963

MATERIAL. $2 \sigma^{7} \sigma^{7}, 1$ (ZMUM 03114$)$, China, Sichuan Prov., SSE of Shimian, S of Zhuma, N29 $02^{\prime} 07^{\prime \prime}$, E102 $28^{\prime} 59^{\prime \prime}$, 2360 m a.s.1., 24.V.2014, leg. I. Belousov \& I. Kabak.

REMARK. This species, originally described from Jiangxi Prov., China [Hoffman, 1963], is quite common and widespread across southern China [Liu, in litt.].

Kronopolites lunatus Likhitrakarn, Golovatch et Panha, 2015

MATERIAL. $1 \bigcirc^{7}, 3$ 우 (ZMUM $\rho 3113$ ), Laos, Xieng Khouang Prov., ca $9 \mathrm{~km}$ NW of Vieng Thong, old secondary tropical 
forest, 870-910 m a.s.1., $\mathrm{N} 20^{\circ} 08.466^{\prime}$, E103²0.099', in logs, 12.XI.2006, leg. A. Abdou \& I.V. Muratov.

REMARKS. This is the only Kronopolites species currently known to occur in Laos [Likhitrakarn et al., 2015]. Above is a third locality, the previous two also being confined to central Laos.

\section{Helicorthomorpha holstii (Pocock, 1895)}

MATERIAL. $10 \sigma^{7} \sigma^{7}, 2$ 우 (ZMUM 03069 ), Japan, Ryukyu Islands, Okinawa Is., Naha-shi, Tomari, 30.XII.1994, leg. M. Kimura.

REMARK. This common East to Southeast Asian species, which is also known as introduced to the southern USA, has long been recorded in the Ryukyus, including Okinawa Island, Japan [e.g. Nakamura \& Korsós, 2010].

\section{Helicorthomorpha luzoniensis (Peters, 1864)}

MATERIAL. $10^{7}, 1$ ( (ZMUM $\rho 3070$ ), Laos, Luang Prabang Prov., near Luang Prabang, Kuangsi Waterfall, ca $370 \mathrm{~m}$ a.s.l., $19^{\circ} 44^{\prime} 50^{\prime \prime} \mathrm{N}, 101^{\circ} 59^{\prime} 33^{\prime \prime} \mathrm{E}, 14 . X I .2015$, leg. I. Melnik.

REMARKS. This species has hitherto been recorded from the Philippines, Indonesia, including New Guinea, as well as China and Cambodia [Golovatch, 2015]. Above is thus the first formal record of $H$. luzoniensis in Laos.

\section{Nedyopus dawydoffiae (Attems, 1953)}

MATERIAL. $1 \sigma^{7}$ (ZMUM 03068 ), Vietnam, Dak Lak Prov., Chu Yang Sin National Park, ca 1000 m a.s.l., upper reaches of Krong Kmak River, $12^{\circ} 23^{\prime} 48^{\prime \prime} \mathrm{N}, 108^{\circ} 20^{\prime} 59^{\prime \prime} \mathrm{E}$, evergreen tropical forest, 30.III-14.IV.2012, leg. D. Fedorenko.

REMARKS. This species has hitherto been known only from two localities in Lam Dong Province. southern Vietnam [Golovatch, 2009a]. Above is the first record of $N$. dawydoffiae in Dak Lak Province, also in southern Vietnam.

\section{Orthomorphella pekuensis (Karsch, 1881)}

MATERIAL. $1 \sigma^{7}$ (SMF), China, Beijing, 6.VII.2013, leg. J. Martens.

REMARKS. This East Asian species is very common, often synanthrope in Korea, Taiwan, Japan and northeastern China, including Beijing which is the type locality.

\section{Oxidus gracilis (C.L. Koch, 1847)}

MATERIAL. $10^{7}$ (MCSNV), China, Guizhou Prov., Libo County, Cave Shu Jia Dong, 17.XI.2006, leg. T. Conci, A. Zorzi \& R. Zorzin; $1 \mathrm{O}^{\mathrm{T}}$ (MCSNV), China, Guizhou Prov., Guanling County, Huajian, Cave Sha Dong, 4.VI.2006, leg. L. Latella \& S. Meggiorini.

REMARK. This ubiquitous, anthropochore species is very common and widespread also throughout China [e.g. Golovatch, 2013a].

Touranella peculiaris Golovatch, 2009

MATERIAL $1 \Im^{r}, 1$ q, 1 subadult $q$ (ZMUM p3072), Vietnam, Lam Dong Prov., Bi Doup - Nui Ba Nature Reserve, 1400-1900 m a.s.1., $12^{\circ} 10^{\prime} \mathrm{N}, 108^{\circ} 40^{\prime} \mathrm{E}$, evergreen tropical forest, 1-22.IV.2008, leg. D. Fedorenko.

REMARKS. The above samples represent strict topotypes [Golovatch, 2009b].

\section{Chamberlinius vietnamicus sp.n.} Figs 1-7.

HOLOTYPE O (MNHN JA 138), Vietnam, Quang Binh Province, Hoan Son village, Cave Hang Cha Ra, $17.768511^{\circ} \mathrm{N}, 105$. $895018^{\circ} \mathrm{E}, 400 \mathrm{~m}$ a.s.1., 21.XI.2011, leg. L. Deharveng \& A. Bedos (Vn11-40).

PARATYPE: 1 juv. fragment (MNHN JA 138), same data, together with holotype.

DIAGNOSIS. Differs from congeners in the uniformly pallid to light yellow coloration, the especially high and mostly slightly upturned paraterga and the shape of the solenophore [cf. Chen et al., 2011b; Nguyen, Korsós, 2011]. sharing only with C. uenoi (Murakami, 1975), from the Ryukyu Islands, Japan, the relatively small size (width $<4.0 \mathrm{~mm}$ ), the absence of a gonopod distofemoral geniculation and the considerably shorter solenomere and solenophore [cf. Murakami, 1975].

NAME. To emphasize the provenance from Vietnam; adjective.

DESCRIPTION. Length of holotype ca $20 \mathrm{~mm}\left(\mathrm{O}^{T}\right)$, width of midbody pro- and metazonae 1.8 and $3.9 \mathrm{~mm}$, respectively $\left(\sigma^{7}\right)$. General coloration in alcohol pallid, only head light yellow (Figs 1-3).

Body polydesmoid, with 20 segments (Figs $1 \& 2$ ). Antennae very long and slender, poorly clavate, slightly extending back behind segment $4\left(\mathrm{O}^{7}\right)$ when stretched dorsally; antennomeres 2-6 subequal in length; interantennal isthmus ca 1.2 as broad as diameter of antennal socket.

In width, head $<<$ collum $<$ segment $2=3=4<5-$ 15; thereafter body gradually tapering towards telson (Figs $1 \& 2$ ). Tegument smooth and shining, dorsum and sterna delicately microgranulate throughout, surface below paraterga a little more coarsely so; in holotype nearly translucent, in paratype opaque. Head densely and regularly setose, only occipital region bare; epicranial line superficial, poorly-developed. Collum tauriform, its fore margin between paraterga a little concave. Dorsum very poorly $\left(\sigma^{7}\right)$ to moderately convex (juv.), devoid of sculture. Paraterga high, mostly set at about upper $1 / 4$ of body height; all paraterga lying below dorsum (especially well so in juv.) and very broad, on collum broadly rounded and subhorizontal, with two minute incisions laterally; on following segments mostly evidently upturned; anterior shoulders nearly straight and directed increasingly caudad; caudolateral corner sharp and increasingly acutangular, increasingly strongly produced behind rear tergal margin on segments 2-19; lateral margin with two distinct, mostly sharp denticles. Paraterga 2 about level to those on collum and segment 3. Calluses indistinct, traceable mainly as narrow, elongated, dorsolateral, margined grooves in caudal halves of poriferous paraterga. Pore formula normal, ozopores small, inconspicuous, dorsal, lying at bottom of callus groove about level to $2^{\text {nd }}$ 


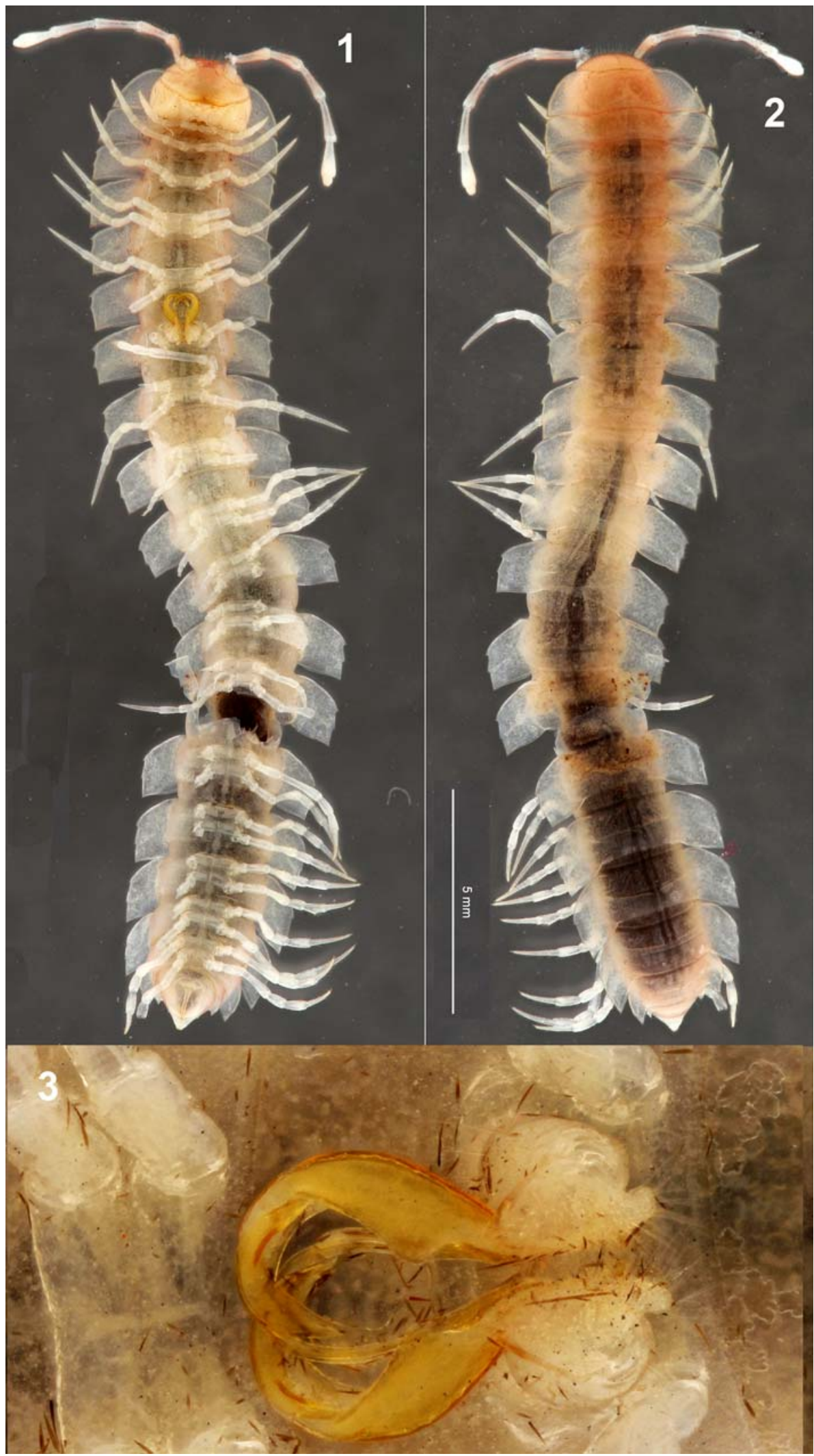

Figs 1-3. Chamberlinius vietnamicus sp.n., holotype: $1 \& 2$ - habitus, dorsal and ventral views, respectively; 3 - gonopods in situ, ventral view. Pictures by J. Brecko, photograph 3 not taken to scale.

Рис. 1-3. Chamberlinius vietnamicus sp.n., голотип: 1 и 2 - общий вид, соответственно сверху и снизу; 3 - гоноподы на месте, снизу. Фотографии J. Brecko, фотография 3 снята без масштаба. 

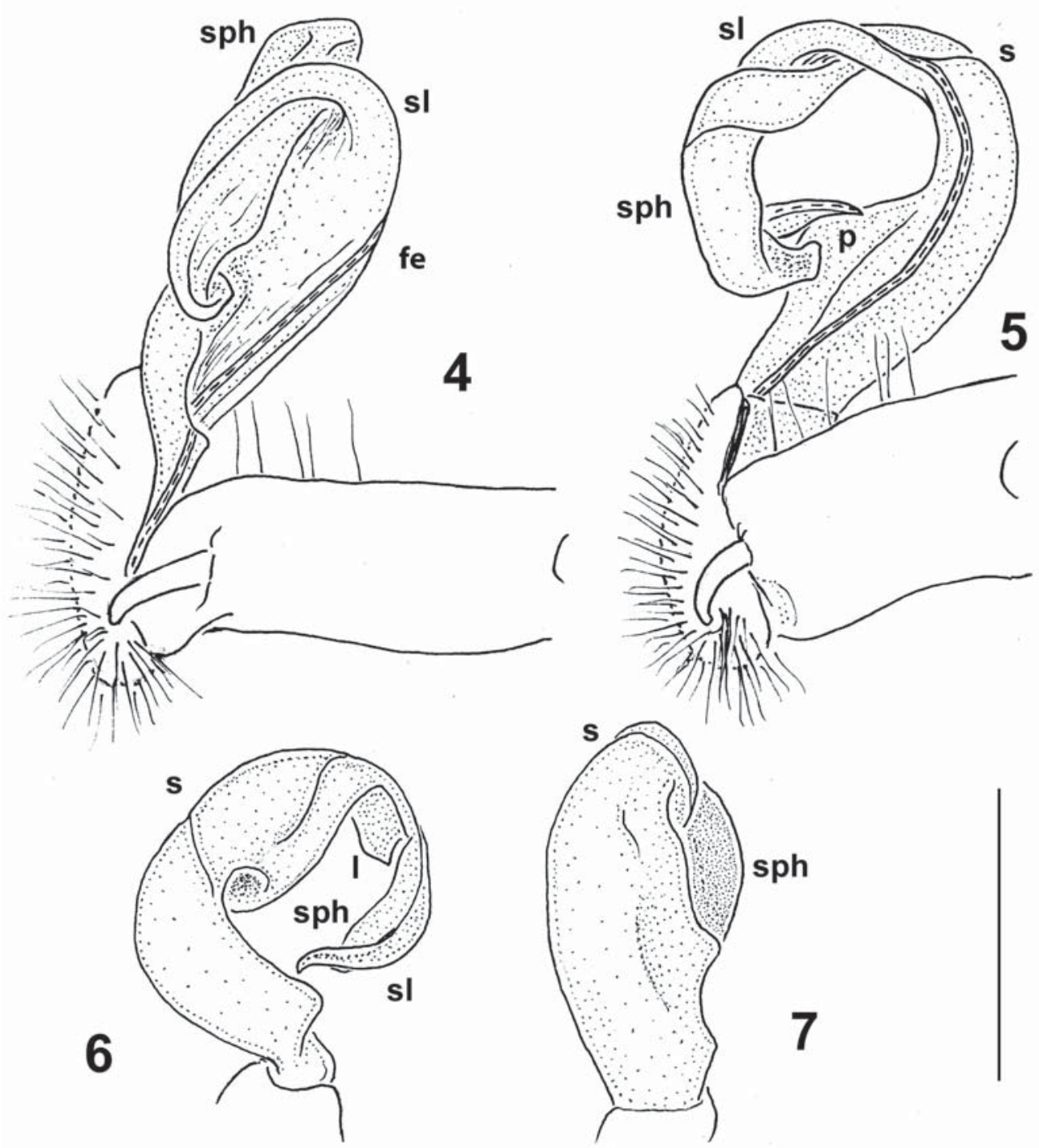

Figs 4-7. Chamberlinius vietnamicus sp.n., holotype, right gonopod, mesal, dorsal, ventral and lateral views, respectively. Scale bar: $0.5 \mathrm{~mm}$.

Рис. 4-7. Chamberlinius vietnamicus sp.n., голотип, правый гонопод, соответственно изнутри, сверху, снизу и сбоку. Масштаб: 0,5 мм.

lateral denticle on paraterga. Transverse metatergal sulcus superficial, visible on segments 5-18, thin, reaching bases of paraterga (Fig. 1). Tergal setae nearly fully abraded, pattern untraceable; individial only seldom retained in a transverse pre-sulcus row, thin, simple, about $1 / 5-1 / 6$ as long as metatergum; on some segments a few shorter setae retained also at caudal margin below paraterga. Stricture dividing pro- and metazonae shallow and rather broad, indistinctly striolate at bottom. Pleurosternal carinae in holotype well-developed rounded, low, well arcuated crests gradually disappearing towards segment 10 , with an evident caudal tooth on segments 3-5. Axial line missing. Epiproct (Figs $1 \& 2$ ) rather long, conical, subtruncate at apex, subapical lateral papillae small and placed rather close to tip. Hypoproct (Fig. 2) subtrapeziform, caudal 1+1 setae missing, not borne on knobs, caudal margin faintly concave.

Neither sternal modifications nor adenostyles, nor tarsal brushes. Sterna flat, nearly bare, cross-impressions weak, especially axial one. Legs long and slender, in holotype ca 1.3-1.4 times as long as midbody height, prefemora not swollen laterally, femora and tarsi subequal, clearly longest, much longer than also subequal coxae, postfemora and tibiae. Each coxa 2 in holotype with an evident distoventral cone carrying a gonopore. Gonopod aperture 8-shaped, its lateral margins slightly elevated, flanked anteriorly by small spiracle-bearing crests.

Gonopods (Figs 4-7) rather simple; in situ crossing each other; coxite very long, slender, subcylindrical, poorly setose distoventrally; telopodite very long and 
rather slender, distinctly curved mesad; prefemoral region nearly $1 / 3$ as long as acropodite, the latter's femoral part (fe) not twisted, but rather well curved, with a moderate, subtriangular, lateral lobe at about midway; fe with a clear, but rather low, subtriangular, midway, lateral lobe (p); fe set off apically from acropodite by a distinct sulcus (s), being devoid of a geniculation; solenomere (sl) a rather thick and long branch, but still slenderer than a similarly strongly curved, subcircular, twisted, slightly shorter and apically subtruncate solenophore (sph), the latter branch subtending an apically acuminate sl and at base showing an evident mesal lobe (l).

REMARKS. The genus Chamberlinius Wang, 1956, has recently been revised [Chen et al., 2011; Nguyen, Korsós, 2011] and shown to comprise five species. Three of them are restricted to Taiwan, one is particularly widespread not only on Taiwan, but also on the Ryukyus, southern Japan, whence still one more endemic congener is known to occur.

Superficially, Chamberlinius is very easy to recognize, as it is nearly unique amongst Paradoxosomatidae in showing paraterga 2 lying level to both the collum and paraterga 3 . In addition, this genus is amongst the rather few which show no sternal modifications between $O^{7}$ coxae 4.

Its occurrence in southern Vietnam is rather surprising, yet this only emphasizes the Oriental roots of the remaining congeners known in Taiwan and southern Japan. The same applies to the entire tribe Chamberliniini the genus belongs to. The present record of a Chamberlinius in Indochina suggests that further congeners may well occur in the region.

\section{Delarthrum quadridentatum sp.n.} Figs 8-22.

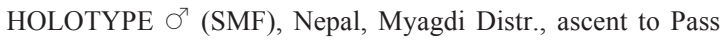
Jaljala, 3000-3200 m a.s.l., 6.IV.1970, leg. J. Martens.

DIAGNOSIS. Differs from other Delarthrum spp. in the presence of four characteristic, membranous, mostly sharp teeth on the solenophore distal to a strong, mesal, parabasal hook.

NAME. To emphasize the presence of four peculiar, mostly subequal, membranous teeth on the solenophore; adjective.

DESCRIPTION. Holotype incomplete, represented by three pieces: anterior part of body (head and segments 1-7, Fig. 8), two midbody segments (Fig. 9) and posterior part of body (last 8 segments, Fig. 10). Length presumably ca $12-13 \mathrm{~mm}\left(\mathrm{O}^{7}\right)$, width of midbody proand metazonae 1.5 and $1.7 \mathrm{~mm}$, respectively $\left(\sigma^{7}\right)$. General coloration in alcohol uniformly light yellowish (Figs 8-13).

Clypeolabral region rather sparsely setose, vertigial one bare; epicranial suture evident (Fig. 11). Antennae short and moderately clavate, in situ slightly extending back behind collum when stretched dorsally $\left(\bigcirc^{7}\right)$; antennomere $2=6>3-5>1>7$; interanten- nal isthmus about 1.2 times as broad as diameter of antennal socket.

In width, collum $<$ segment $2=3<$ head $=$ segment $4<5-15(16)$; thereafter body gradually tapering towards telson. Tegument smooth and slightly shining, prozonae finely shagreened, metazonae microalveolate, surface below paraterga also microgranulate. Collum broadly rounded laterally. Postcollum paraterga poorly-developed, low, mostly set at about half of body height; delimited by a complete distinct sulcus only dorsally, in about caudal 1/3 also ventrally (Fig. 14); caudal corner mostly sharpened, but never drawn back behind rear tergal margin, only paraterga 2 clearly produced forward. Poriferous calluses somewhat thicker than poreless ones; ozopores lateral, lying inside an ovoid groove (Fig. 14). Transverse metatergal sulcus superficial, visible on segments 5-17(18), not reaching bases of paraterga, absent from penultimate segment. Tergal setae fully abraded, pattern untraceable. Stricture dividing pro- and metazonae thin and deep, finely striolate at bottom down to level of paraterga. Pleurosternal carinae an evident, but low squarish lobule only on segment 2, thereafter an increasingly low longitudinal bulge traceable until about midbody segment. Axial line missing. Epiproct (Figs 10, 13 \& 15) rather long, clearly flattened dorsoventrally, conical, subtruncate at apex, subapical lateral papillae small and placed close to tip. Hypoproct (Fig. 16) semi-circular, caudal $1+1$ setae well separated, not borne on knobs.

Sterna sparsely setose, without modifications except for a small, but distinct, rounded, setose, tongueshaped lobe between coxae 4 (Fig. 17); cross-impressions faint. Adenostyle on femur 1 very distinct (Fig. 18). Legs short and rather stout $\left(\sigma^{7}\right)$, only $0.9-1.0$ times as long as midbody height $\left(\sigma^{7}\right)$, prefemora not swollen laterally; in length, femora $>$ prefemora $>$ coxae $=$ postfemora $=$ tarsi; claw slightly curved, ca $2 / 3$ as long as tarsus. Tibial and tarsal brushes absent only from a few last leg-pairs $\left(O^{7}\right)$.

Gonopods (Figs 19-22) rather simple; in situ held parallel to each other; coxite long, slender, subcylindrical, poorly setose distoventrally; telopodite very long and rather slender, suberect; prefemoral (= densely setose) region ca $1 / 3$ as long as acropodite, the latter's femoral part (fe) not twisted, only moderately broadened at midlength, set off from solenophore (sph) by a mainly dorsal sulcus (s); fe ca 1.5 times as long as $\mathbf{s p h}$, the latter with a strong, mesal, parabasal hook (h) and three membranous, sharp, subequal teeth more distally: a mesal, b lateral and c apical; solenomere (sl) almost as long as $\mathbf{s p h}$, flagelliform, its tip exposed near base of c.

REMARKS. At present, the large, mostly Himalayan genus Delarthrum Attems, 1936 encompassses as many as 55 species [Golovatch, 2014, 2015; Golovatch, Wesener, 2016]. The new species is distinguished primarily by its solenophore conformation that shows four, largely membranous, sharp, characteristic teeth, coupled with a slender and flagelliform solenomere. 

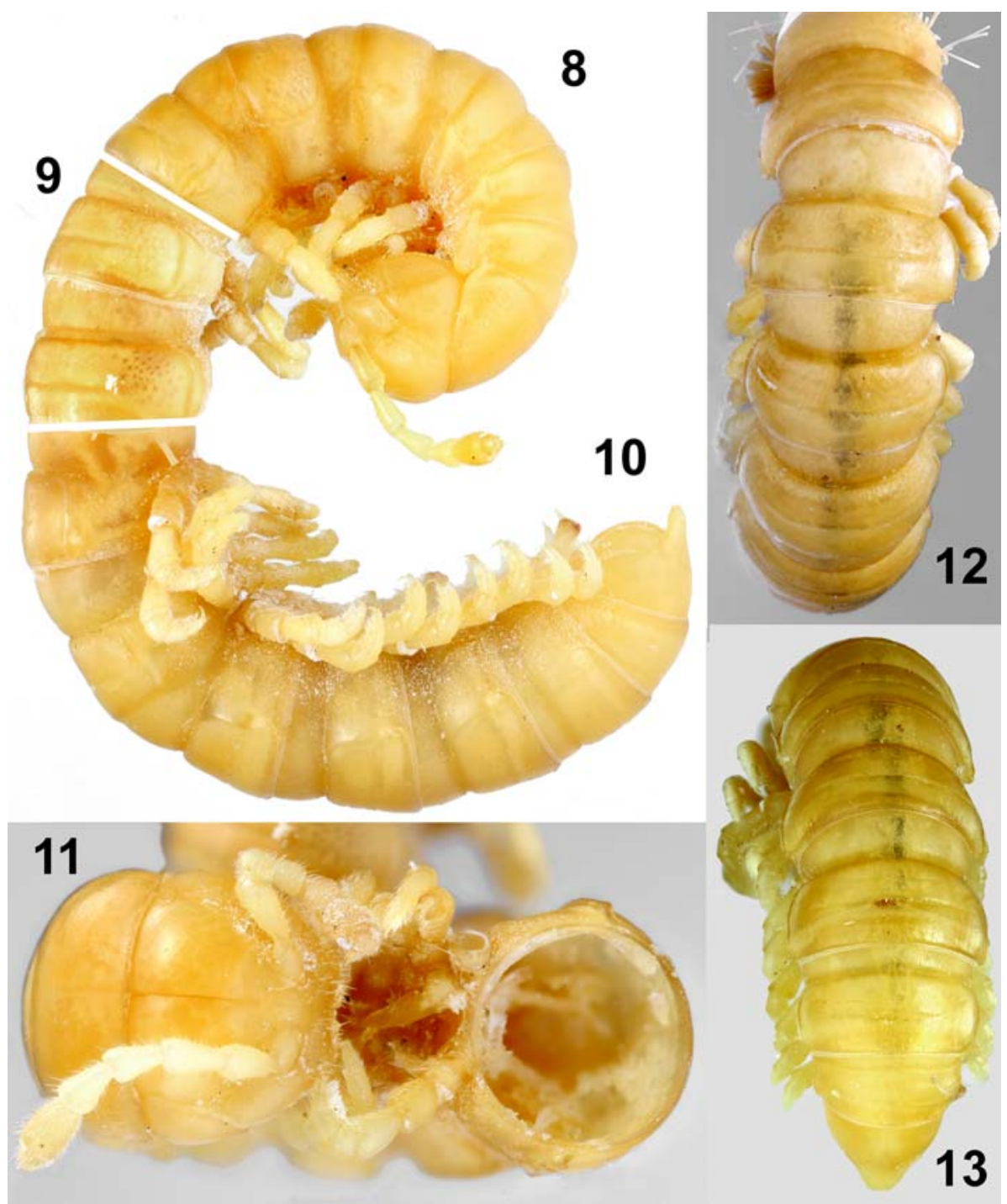

Figs 8-13. Delarthrum quadridentatum sp.n., holotype: $8 \& 11$ - anterior part of body, lateral and ventral views, respectively; 9 two midbody segments, lateral view; 10, $12 \& 13$ - posterior part of body, lateral, dorsal and dorsal views, respectively. Pictures by K. Makarov, not taken to scale.

Рис. 8-13. Delarthrum quadridentatum sp.n., голотип: 8 и 11 - передняя часть тела, соответственно сбоку и снизу; 9 - два среднетуловищных сегмента, сбоку; 10, 12 и 13 - задняя часть тела, соответственно сбоку, сверху и сверху. Фото К. Макарова, сняты без масштаба.

\section{Touranella pilosa sp.n.}

Figs 23-33.

HOLOTYPE OT (SMF), Nepal, Sankhua Sabha Distr., above Pahakhola, 2600-2800 m a.s.l., Quercus semecarpifolia, Rhododendron scrub and trees, 31.V-3.VI.1988, leg. J. Martens \& W. Schawaller.

PARATYPES: 2 우 (SMF), same data, together with holotype.

DIAGNOSIS. Differs from other Touranella spp. by the very densely and irregularly pilose collum and following metaterga, coupled with the presence of as many as three processes on an especially strongly abbreviated gonopod femorite. See also Key below.

NAME. To emphasize the abundantly pilose/setose collum and following metaterga.

DESCRIPTION. Length ca $12\left(\sigma^{7}\right)$ or $13-14 \mathrm{~mm}$ (†), width of midbody pro- and metazonae 1.2 and 1.4 $\left(\sigma^{7}\right)$ or $1.45-1.55$ and $1.6-1.7 \mathrm{~mm}(+)$, respectively. General coloration in alcohol whitish grey to light yellowish-brown, with an indistinct cingulate pattern of light yellow-brown metazonae or only metaterga alternating with lighter, nearly whitish prozonae (Figs 2328); antennomeres 6 and 7 contrasting increasingly dark brown (Figs 25 \& 26); distal podomeres sometimes faintly infuscate, very light brown (Fig. 25).

Clypeolabral region densely, vertigial one sparsely, setose; epicranial suture superficial, more evident on vertex (Fig. 26). Antennae short and moderately clavate, in situ slightly extending back behind segment 2 $\left(\sigma^{7}\right)$ or collum $(+)$ when stretched dorsally; antennomere $2=6>3-5>1=7$; interantennal isthmus about 1.2 times as broad as diameter of antennal socket.

In width, head $<$ segment $2=3<$ collum $<$ segment 


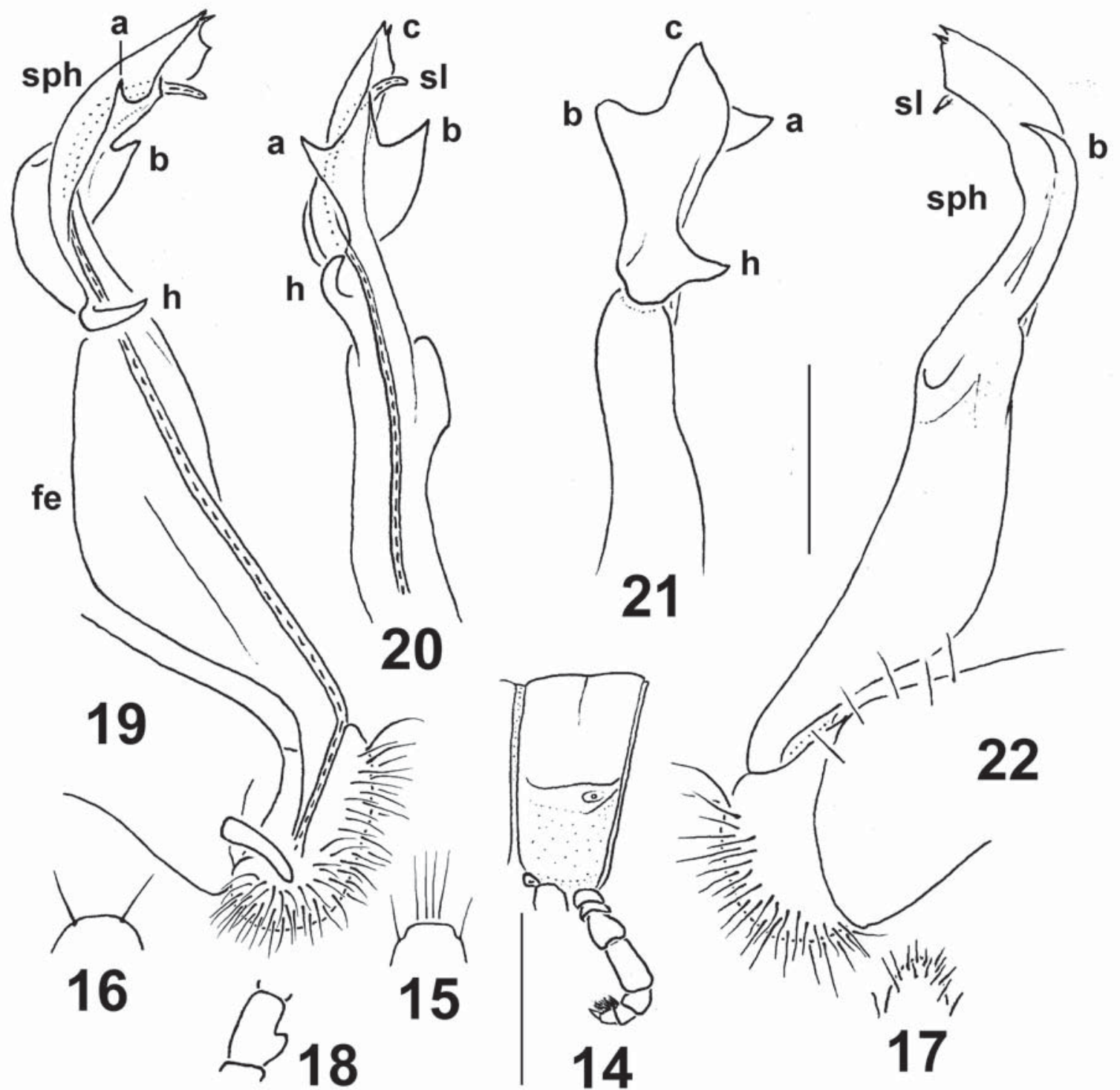

Figs 14-22. Delarthrum quadridentatum sp.n., holotype: 14 - midbody pore-bearing segment, lateral view; 15 - tip of epiproct, dorsal view; 16 - hypoproct, ventral view; 17 - sternal lobe between coxae 4, caudal view; 18 - femur 1 , lateral view; 19-22 - left gonopod, mesal, ventral, subdorsal and lateral views, respectively. Scale bars: 1.0 (14), 0.5 (15-18) and 0.2 mm (19-22).

Рис. 14-22. Delarthrum quadridentatum sp.n., голотип: 14 - среднетуловищный несущий пору сегмент, сбоку; 15 - кончик эпипрокта, сверху; 16 - гипопрокт, снизу; 17 - стернальная пластина между тазиками 4, сзади; 18 - бедро 1, сбоку; 19-22 левый гонопод, соответственно изнутри, снизу, почти сверху и сбоку. Масштаб: 1,0 (14), 0,5 (15-18) и 0,2 мм (19-22).

$4<5-16$; thereafter body gradually tapering towards telson. Tegument mostly smooth and slightly shining, prozonae finely shagreened, metazonae microalveolate, surface below paraterga also microgranulate, sometimes faintly rugulose (Figs 23-27). Collum broadly rounded laterally. Postcollum paraterga poorly-developed, low, mostly set at about $1 / 3$ of body height; delimited by a complete distinct sulcus only dorsally, in about caudal $1 / 3$ to half also ventrally (Fig. 27); caudal corner mostly sharpened, but drawn back behind rear tergal margin only starting with segment 16; only paraterga 2 clearly produced forward. Poriferous calluses somewhat thicker than poreless ones, each with 4-5 setigerous knobs laterally; ozopores lateral, invisible from above, lying inside a slight longitudinal groove removed forward ca $1 / 4$ off caudal paratergal corner (Fig. 29). Transverse metatergal sulcus distinct on segments 5-18, not reaching bases of paraterga, absent from segment 19 . Tergal setae very abundantly and irregularly clothing collum and all following metaterga, simple, mostly up to $1 / 3$ as long as metatergite, largely not borne on knobs (Figs 23-27). Stricture dividing pro- and metazonae thin and deep, finely striolatepunctate at bottom down to level of paraterga. Pleurosternal carinae an evident, but low squarish lobule only on segment 2 , thereafter an increasingly low longitudinal bulge traceable until about segment $7\left(\sigma^{7}\right)$. Axial line missing. Epiproct (Figs 23, 26 \& 28) rather long, clearly 

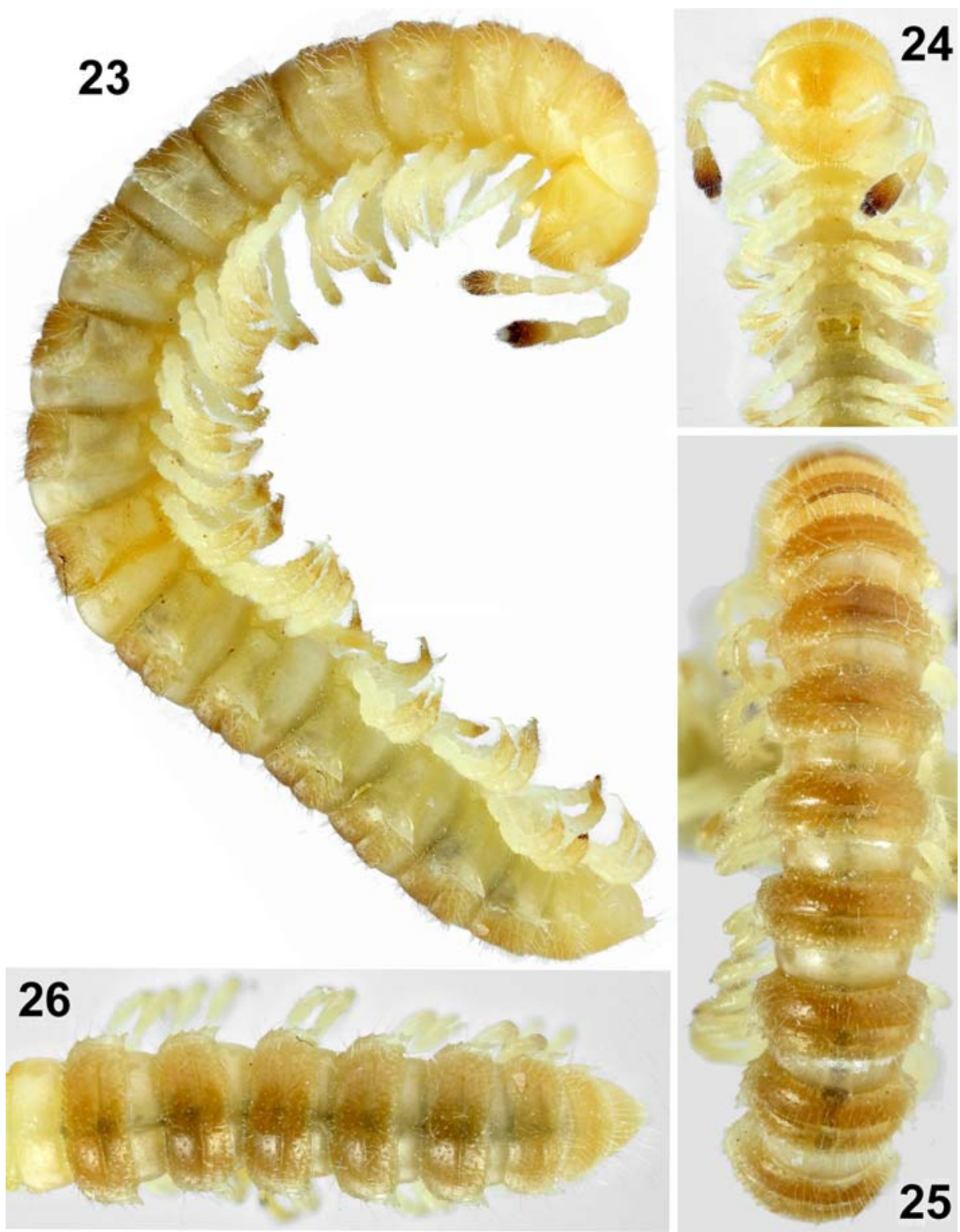

Figs 23-26. Touranella pilosa sp.n., holotype: 23 - habitus, lateral view; 24 - anterior part of body, ventral view; 25 - middle part of body, dorsal view; 26 - posterior part of body, dorsal view. Pictures by K. Makarov, not taken to scale.

Рис. 23-26. Touranella pilosa sp.n., голотип: 14 - общий вид, сбоку; 24 - передняя часть тела, снизу; 25 - средняя часть тела, сверху; 26 - задняя часть тела, сверху. Фото К. Макарова, сняты без масштаба.

flattened dorsoventrally, conical, subtruncate at apex, subapical lateral papillae small and placed quite close to tip. Hypoproct (Fig. 29) roundly subtrapeziform, caudal $1+1$ setae well separated, not borne on knobs.

Sterna sparsely setose, cross-impressions weak, without modifications other than a small, but distinct, rounded, setose, tongue-shaped lobe between $\sigma^{7}$ coxae 4 (Fig. 30). Adenostyle on $\sigma^{7}$ femur 1 distinct. Legs moderately long and rather slender, ca 1.1-1.2 $\left(\sigma^{7}\right)$ or 0.9-1.0 times ( $(+)$ as long as midbody height, $\sigma^{7}$ prefemora moderately swollen laterally; in length, femora = tarsi $>$ prefemora $>$ coxae $=$ postfemora $=$ tibiae; claw slightly curved (Fig. 27). Tarsal brushes present only on legs 1 and $2\left(O^{7}\right)$.
Gonopods (Figs 31-33) complex; in situ held subparallel to each other; coxite rather short, subcylindrical, poorly setose distally; telopodite very long and slender, suberect; prefemoral (= densely setose) region ca $1 / 3$ as long as acropodite, the latter's femoral part (fe) particularly strongly abbreviated, almost fully suppressed, supplied by a short, spoon-shaped, lateral (fp), an almost thrice as long, slightly sigmoid, slender, subacuminate, dorsal (dp) and a lanceolate, clearly acuminate, ventral process (vp), the latter about half as long as entire postfemoral part. Solenomere shaft (sl) strong, erect, mostly sheathed by a delicate membranous solenophore (sph); distal quarter of sph branching into a large, round, apical, membranous lobe (lo) and a slen- 

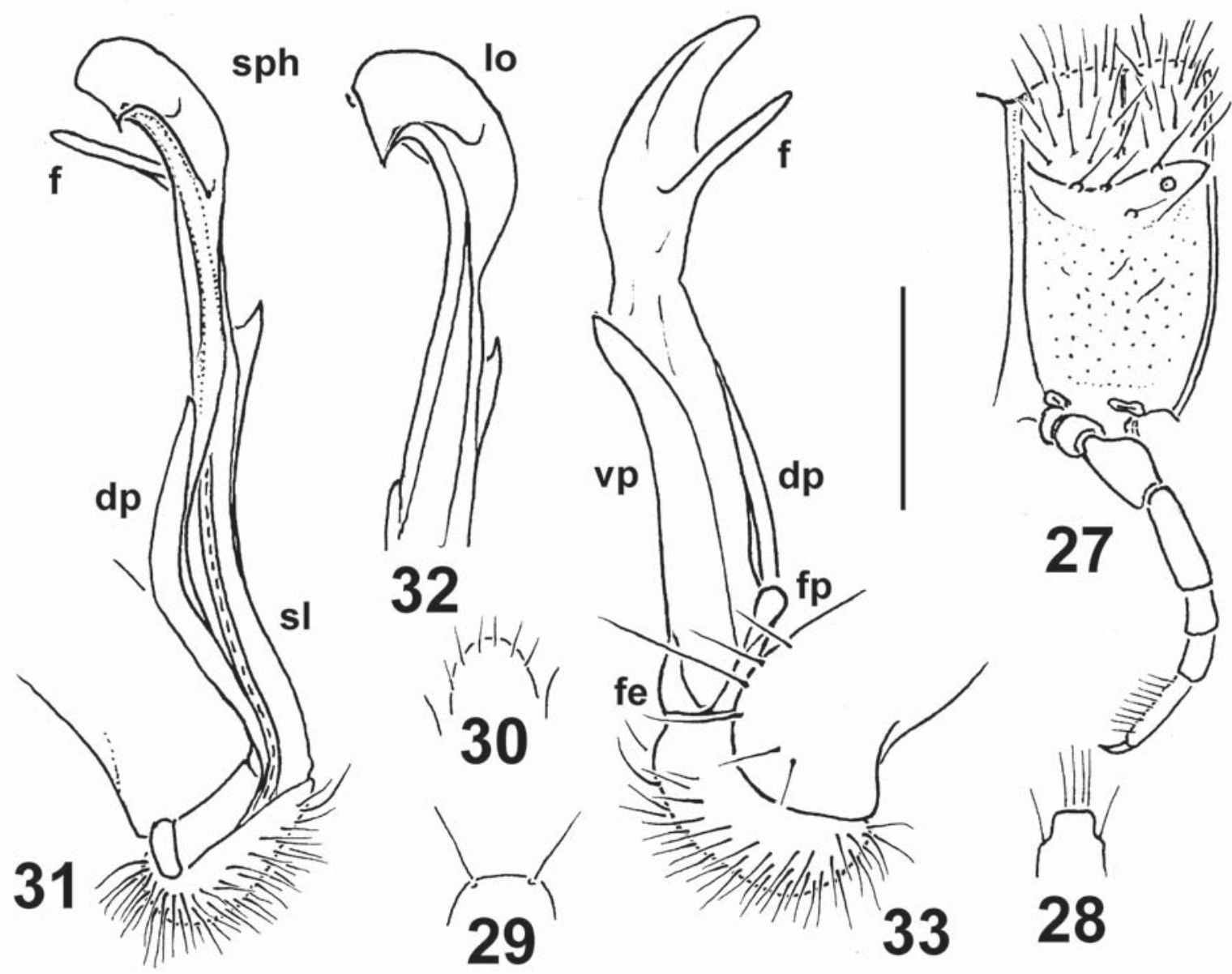

Figs 27-33. Touranella pilosa sp.n., holotype: 27 - segment 10, lateral view; 28 - tip of epiproct, dorsal view; 29 - hypoproct, ventral view; 30 - sternal lobe between coxae 4, caudal view; 31-33 - left gonopod, mesal, ventral and lateral views, respectively. Scale bar: $0.6(27-30)$ and $0.2 \mathrm{~mm}(31-33)$.

Рис. 27-33. Touranella pilosa sp.n., голотип: 14 - сегмент 10, сбоку; 28 - кончик эпипрокта, сверху; 29 - гипопрокт, снизу; 30 - стернальная пластина между тазиками 4, сзади; 31-33 - левый гонопод, соответственно изнутри, снизу и сбоку. Масштаб: $0,6(27-30)$ и 0,2 мм (31-33).

der subapical finger (f), basal part of lo on mesal face sheathing and concealing sl tip.

REMARKS. The genus Touranella Attems, 1937, has hitherto been known to encompass five species: $T$. gracilis Attems, 1937, the type-species from Vietnam, T. himalayaensis Golovatch, 1994, from Nepal, as well as T. peculiaris Golovatch, 2009, T. hirsuta Golovatch, 2009 and T. cattiensis Golovatch et Semenyuk, 2010, all three again from Vietnam [Attems, 1937, 1938; Golovatch, 1994, 2009a, b; Golovatch, Semenyuk, 2010]. In addition to T. pilosa sp.n., the following three species show similarly densely pilose/ setose collum and following metaterga: $T$. gracilis, $T$. himalayaensis and T. hirsuta, although in the latter congener the tergal setae are borne on small rounded bosses and mostly arranged in six regular transverse rows. In addition, T. pilosa sp.n. shares a similar, particularly strongly abbreviated, almost vestigial gonopod femorite carrying a basal process only with $T$. gracilis and $T$. himalayaensis, but neither of the latter two species has as many as three processes.
The following key can be proposed to incorporate the new species:

1(2) Collum and following metaterga very densely hirsute (Fig. 23-27)

2(1) Collum and following metaterga not so densely hirsute, tergal setae being much fewer. Vietnam ....................... 9

3(4) Postcollum metaterga mostly with six transverse regular rows of tergal setae borne on small bosses. Gonopod femorite devoid of processes. Vietnam ......... T. hirsuta

4(3) Postcollum metaterga very densely and irregularly hirsute (Fig. 27). Gonopod femorite supplied at least with one process

5(6) Gonopod femorite especially short, carrying as many as three processes: fp, vp and dp (Figs 31-33)

T. pilosa sp.n

6(5) Gonopod femorite not so short, carrying only one distolateral process

7(8) Gonopod femoral process relatively long, about half as long as acropodite distal to prefemoral (= densely setose) part. Nepal T. himalayaensis

8(7) Gonopod femoral process much shorter. Vietnam ... T. gracilis 

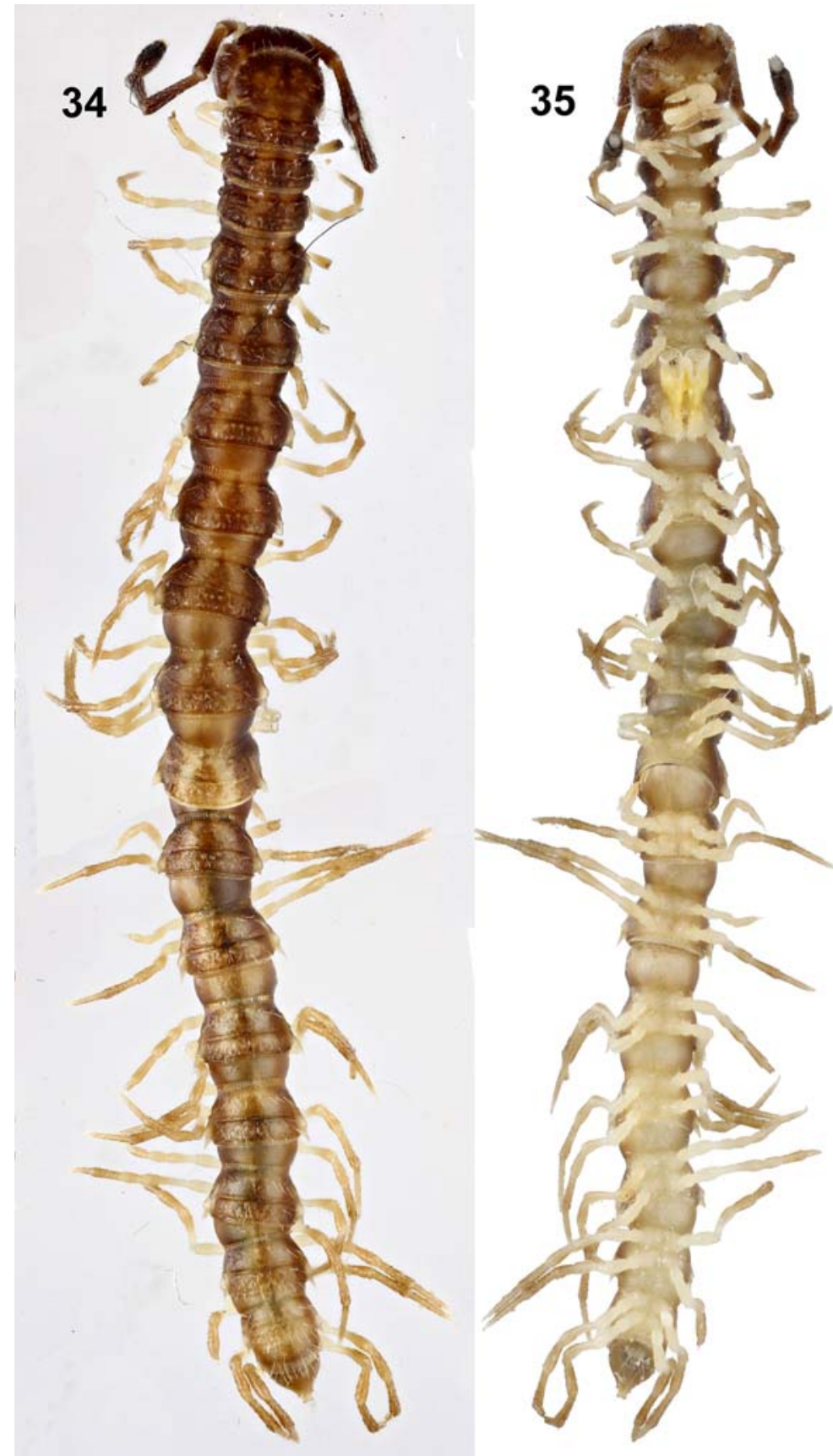

Figs 34 \& 35. Habitus of Pocockina jaegeri sp.n., holotype, dorsal and ventral views, respectively. Pictures by K. Makarov, not taken to scale.

Рис. 34 и 35. Общий вид Pocockina jaegeri sp.n., голотип, соответственно сверху и снизу. Фото К. Макарова, сняты без масштаба.
9(10) Gonopod femorite not so strongly abbreviated, because a free solenomere branching off at about gonopod telopodite midway T. peculiaris

10(9) Gonopod femorite much more strongly abbreviated, because a free solenomere branching off at about basal $1 / 5$ of gonopod telopodite T. cattiensis

\section{Pocockina jaegeri sp.n.}

Figs 34-44.

HOLOTYPE $0^{7}$ (SMF), Myanmar, S Chin State, above Kam-

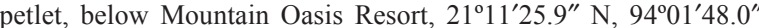
E, secondary tropical forest along stream, 15.V.2014, leg. P. Jäger \& J. Martens.
PARATYPES: 1 q, 1 fragm. $\sigma^{7}$ (head and segments $1-8$ ), 1 fragm. o (head and segments 1-9), 3 fragments (posterior portions of body) (SMF), $1 \mathrm{O}^{7}$ (ZMUM $\rho 3106$ ), same data, together with holotype.

DIAGNOSIS. Differs from both other Pocockina spp. known to date, $P$. pilifera (Pocock, 1895), the type-species from Myanmar [Jeekel, 1965], and P. schawalleri Golovatch, 2016, from eastern Nepal [Golovatch, 2016], primarily by the cup-shaped gonopods. See also Key below.

NAME. Honours Peter Jäger (SMF), one of the collectors.

DESCRIPTION. Length ca $13-15\left(\bigcirc^{7}\right)$ or $16 \mathrm{~mm}$ (+), width of midbody pro- and metazonae $0.9-1.0$ and 


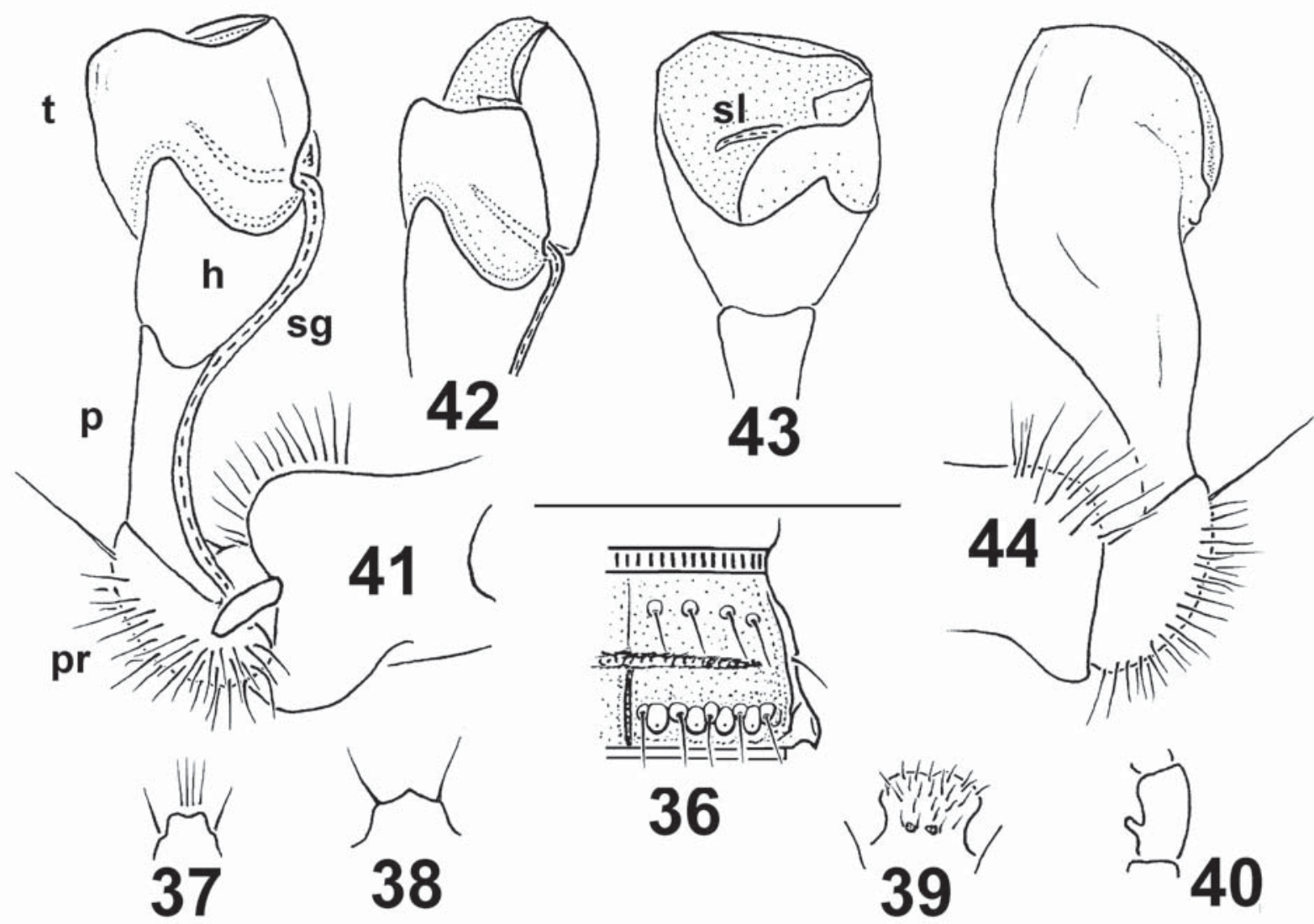

Figs 36-44. Pocockina jaegeri sp.n., holotype: 36 — right half of segment 10, dorsal view; 37 — tip of epiproct, dorsal view; 38 hypoproct, ventral view; 39 - sternal lobe between coxae 4, caudal view; 40 - femur 1, lateral view; 41-44 - right gonopod, mesal, dorsal, ventral and lateral views, respectively. Scale bar: $1.0(36-40)$ and $0.5 \mathrm{~mm}(41-44)$.

Рис. 36-44. Pocockina jaegeri sp.n., голотип: 36 - правая половина сегмента 10, сверху; 37 - кончик эпипрокта, сверху; 38 гипопрокт, снизу; 39 - стернальная пластина между тазиками 4, сзади; 40 - бедро 1, сбоку; 41-44 - правый гонопод, соответственно изнутри, сверху, снизу и сбоку. Масштаб: 1,0 (36-40) и 0,5 мм (41-44).

1.1-1.2 $\left(\sigma^{7}\right)$ or 1.7-1.9 mm $(+)$, respectively, but head broadest, $1.2-1.3\left(\sigma^{7}\right)$ or $2.0 \mathrm{~mm}(+)$ wide. Holotype ca $15 \mathrm{~mm}$ long, 1.0 and $1.2 \mathrm{~mm}$ wide on midbody proand metazonae, respectively; head $1.3 \mathrm{~mm}$ wide. General coloration in alcohol marbled brown to light greyish, anterior half of body infuscate brown compared to a lighter posterior half, dorsum with a rather distinct and characteristic pattern of lighter, hourglass-shaped spots on postcollum pro- and metaterga, as well as of lighter caudal halves of paraterga and of epiproct tip (Fig. 34). Antennae uniformly dark brown, but tip whitish. Venter and several basal podomeres greyish, legs largely slightly infuscate, light brown distally (Figs 34 \& 35).

Head densely setose nearly throughout; epicranial suture evident, especially deep on vertex (Fig. 35). Antennae very long and moderately clavate, in situ slightly extending back behind segment $5\left(\mathrm{O}^{7}\right)$ or $3(+)$ when stretched dorsally; antennomere $3>4=5=6>2$ $>1=7$; interantennal isthmus ca 1.2 times as broad as diameter of antennal socket.

Body moniliform (Figs 34 \& 35). In width, segment $3=4<$ collum $=2<$ segment $5-16<$ head. Tegument mostly uneven, largely roughly tuberculate, shining, only prozonae and surface below paraterga shagreened. Collum crescent-shaped, fore margin broadly rounded, caudal corner of paraterga acute and narrowly rounded; $8+8$ long setae at fore and lateral margins, several similar setae centrally and at caudal edge; setae typically borne on small knobs. Postcollum metaterga with three transverse, rather regular rows of similar setigerous knobs: $3(4)+3(4)$ in one fore (= pre-sulcus) row, followed by $3-5+3-5$ and $4-5+4-5$ in two, strongly adjacent, even more distinct, posterior (= post-sulcus) rows; number of setae per row gradually increased and stabilized to $4+4,5+5$ and $5+5$ towards posterior half of body; setae long, simple, about $1 / 3$ as long as metatergite (Figs 34-36). Paraterga poorly-developed, low, mostly set at ca $1 / 3$ of body height; delimited by a complete distinct sulcus only dorsally, only in about caudal 1/3 also ventrally; caudal corner mostly acute and sharpened, increasingly clearly drawn back behind rear tergal margin starting with segment 13 . Poriferous calluses thicker than poreless ones, each with a setigerous knob laterally near midway of paratergite; ozopores lateral, invisible from above, lying inside an ovoid pit in front of caudal paratergal corner (Figs 3436). Already collum with a slight, transverse, median 


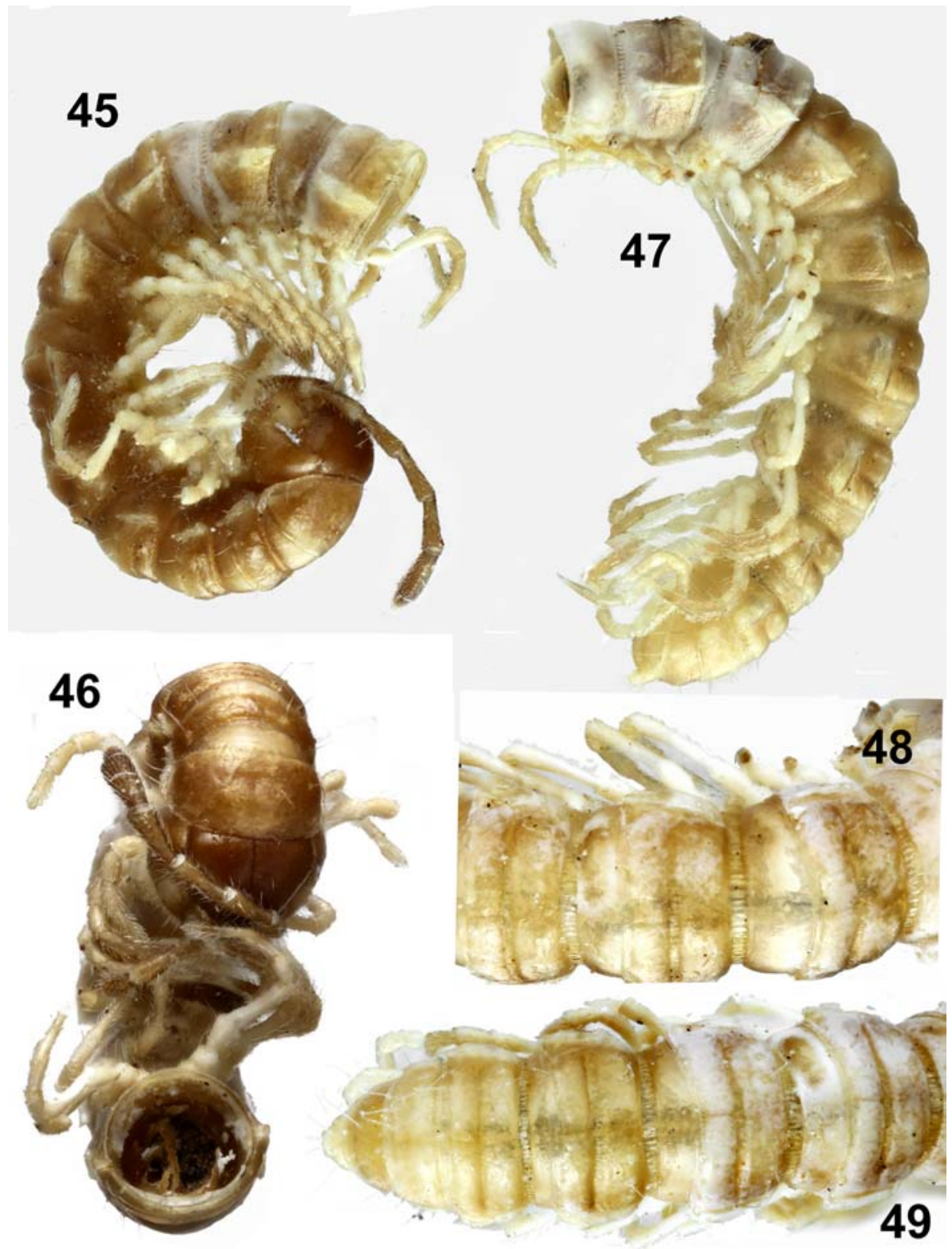

Figs 45-49. Tetracentrosternus martensi sp.n., holotype: 45 \& 46 - anterior part of body, lateral and ventral views, respectively; 47 \& 49 - posterior part of body, lateral and dorsal views, respectively; 48 - middle part of body, dorsal view. Pictures by K. Makarov, not taken to scale.

Рис. 45-49. Tetracentrosternus martensi sp.n., голотип: 45 и 46 - передняя часть тела, соответственно сбоку и снизу; 47 и 49 задняя часть тела, соответственно сбоку и сверху; 48 - средняя часть тела, сверху. Фото К. Макарова, сняты без масштаба.

impression, followed by a deep transverse sulcus on metaterga 2-18; sulci reaching bases of paraterga 5 18, usually ribbed at bottom (Fig. 36), absent from segment 19. Stricture dividing pro- and metazonae broad and deep, clearly ribbed at bottom down to somewhat below paraterga. Pleurosternal carinae an evident round lappet only on segment 2 . Axial line from nearly missing to evident, in the latter case best visible on post-sulcus halves of metaterga (Fig. 36). Epiproct (Figs 34, 35, 37) rather long, clearly flattened dorsoventrally, conical, faintly concave at apex, subapical lateral papillae small, but evident. Hypoproct (Fig. 38) subpentagonal, caudal 1+1 setae well separated, not borne on knobs.
Sterna moderately setose, cross-impressions weak, without modifications other than a prominent, rounded, setose, apically faintly concave, tongue-shaped lobe between $\sigma^{7}$ coxae 4 (Fig. 39). Adenostyle on $\sigma^{7}$ femur 1 distinct (Fig. 40). Legs very long and slender, > 2.5 $\left(\sigma^{7}\right)$ or ca 1.5 times $(+)$ as long as midbody height, $\sigma^{7}$ prefemora not swollen laterally; in length, femora $>$ tarsi $>$ tibiae $>$ prefemora $=$ postfemora $>$ coxae; claw slightly curved (Figs $34 \&$ 35). Tarsal brushes present only on legs $1\left(\mathrm{O}^{\text {T }}\right)$.

Gonopods (Figs 35, 41-44) cup-shaped, in situ held subparallel to each other (Fig. 35); coxite short, about half as long as telopodite, subcylindrical, clearly setose 
distolaterally; telopodite suberect, subdivided into four distinct, subequally high regions: a prefemoral (= densely setose) part (pr), a parabasal, narrowed, pedicelshaped part (p), a chitinous holder part (h) and a thin membranous cup top (t). Three latter parts not separated from each other by evident transverse sulci only on lateral face, none of them being twisted because seminal groove runs entirely on mesal side of telopodite until passing onto a rather short free solenomere (sl). Both $\mathbf{h}$ and $\mathbf{t}$ forming a solenophore showing no visible traces of stiff setiform filaments; sl starting at the very base of $\mathbf{t}$ to become squeezed on dorsal side between t's petal-shaped folds.

REMARKS. The genus Pocockina Jeekel, 1965, has hitherto been known to contain two species only: $P$. pilifera (Pocock, 1895), the type-species coming from Palon in Pegu and from Yangon (= Rangoon), southern Myanmar [Pocock, 1895; Jeekel, 1965], and P. schawalleri Golovatch, 2016, from central Nepal [Golovatch, 2016]. In contrast, P. jaegeri sp.n. derives from the south of Chin State, western Myanmar. The following key can be proposed for their separation.

1(2) Tergal surface nearly smooth, most of metaterga with only two transverse rows of setae not borne on knobs .. $P$. pilifera

2(1) Tergal surface rough, most of metaterga with three transverse rows of setae, at least caudal row being borne on evident knobs

3(4) Epiproct tip unusually broad and strongly flattened. Gonopod solenophore strongly sigmoid, with several stiff setiform filaments. Nepal ............... P. schawalleri

4(3) Epiproct quite usual (Figs 34, 35 \& 37). Gonopod solenophore cup-shaped, devoid of stiff setiform filaments (Figs 35, 41-44). Myanmar ........ P. jaegeri sp.n.

\section{Tetracentrosternus martensi sp.n.}

Figs 45-57.

HOLOTYPE OT (SMF), Myanmar, S Chin State, above Kampetlet, below Mountain Oasis Resort, $21^{\circ} 11^{\prime} 43.6^{\prime \prime} \mathrm{N}, 94^{\circ} 02^{\prime} 1.1^{\prime \prime} \mathrm{E}$ $1550 \mathrm{~m}$ a.s.1., secondary tropical forest along stream, 17.V.2014, leg. P. Jäger \& J. Martens.

PARATYPES: $1 \sigma^{7}$ fragment (head and segments 1-7), 2 우 (SMF), Myanmar, S Chin State, above Kampetlet, below Mountain Oasis Resort, $21^{\circ} 11^{\prime} 25.9^{\prime \prime} \mathrm{N}, 94^{\circ} 01^{\prime} 48.0^{\prime \prime} \mathrm{E}$, secondary tropical forest along stream, 15.05.2014, leg. P. Jäger \& J. Martens.

DIAGNOSIS. Differs from all three other Tetracentrosternus spp. known to date, T. subspinosus (Pocock, 1895), the type-species from Myanmar [Hoffman, 1963; Jeekel, 1965], T. hoffmani Golovatch, 2013 , from Yunnan Province, China [Golovatch, 2013a], and T. theelorsuensis Likhitrakarn, Golovatch et Panha, 2013, from Tak Province, western Thailand [Likhitrakarn et al., 2013], primarily by the especially strongly sigmoid, subgeniculate gonopod telopodite. See also Key below.

NAME. Honours Jochen Martens (Mainz, Germany), one of the collectors.

DESCRIPTION. Length ca $12\left(\sigma^{7}\right)$ or $13 \mathrm{~mm}\left({ }_{+}\right)$, width of midbody pro- and metazonae $0.8-0.9$ and $1.0-1.1\left(O^{7}\right)$ or 1.3 and $1.5 \mathrm{~mm}(+)$, respectively. Holotype ca $12 \mathrm{~mm}$ long, 0.8 and $1.0 \mathrm{~mm}$ wide on midbody pro- and metazonae, respectively. General coloration in alcohol marbled light brown to light greyish, anterior half of body infuscate brown compared to a lighter posterior half; dorsum with a rather vague pattern of lighter, nearly pallid, broad, axial stripe both on proand metazonae, as well as of lighter, nearly pallid venter, epiproct and legs, only the latter's distal podomeres sometimes increasingly infuscate, light brownish (Figs 45-49). Antennae increasingly infuscate, dark brown, but tip whitish.

Clypeolabral region densely setose, vertigial one with 1+1 long setae; epicranial suture evident, especially deep on vertex (Figs $45 \& 46$ ). Antennae short and moderately clavate, in situ slightly extending back behind midlength of segment $2\left(O^{7}\right)$ or collum $(+)$ when stretched dorsally; antennomeres 2-6 subequal in length; interantennal isthmus ca 1.2 times as broad as diameter of antennal socket.

In width, collum $<$ segments $2-4<$ head $<5-16$; thereafter body gradually tapering towards telson. Tegument largely smooth and shining, prozonae shagreened, surface below paraterga microgranulate. Collum with three transverse rows of long simple setae: $3-4+3-4$ at fore edge, $1+1$ in the middle and $3+3$ at caudal margin; paraterga very small, caudal corner subrectangular and narrowly rounded. Postcollum paraterga small, set low at about midheight of metazonae, only in segment 2 drawn both anteriorly and posteriorly, following paraterga mostly acute caudally, pointed to nearly so, but slightly drawn behind rear tergal margin only in segments 16-19; calluses demarcated by a complete distinct sulcus only dorsally, only in about caudal $1 / 3$ by a less distinct sulcus also ventrally; poriferous calluses thicker than poreless ones (Figs 45-51). Metaterga with two transverse regular rows of setae, pattern being $3+3$ in fore (= pre-sulcus) row, followed by $4-5+4-5$ setae borne on oblong knobs in posterior (= post-sulcus) row; number of setae in caudal row gradually increased and stabilized to $5+5$ only towards a few posteriormost segments; setae long, simple, about 1/3$1 / 2$ as long as metatergite, often abraded, traceable also at least as $1+1$ insertion points at about fore $1 / 3$ of postcollum paraterga (Figs 45-51). Transverse sulci present on metaterga 5-18, almost reaching bases of paraterga, usually wide and striolate at bottom (Figs 50 $\&$ 51). Stricture dividing pro- and metazonae broad and deep, clearly ribbed at bottom down to below paraterga. Pleurosternal carinae an evident round lappet only on segment 2 . Axial line from nearly missing to evident, in the latter case best visible on post-sulcus halves of metaterga (Fig. 51). Epiproct (Figs 49 \& 52) rather long, clearly flattened dorsoventrally, conical, subtruncate at apex, subapical lateral papillae small, but evident. Hypoproct (Fig. 53) subtrapeziform, caudal $1+1$ setae well separated, borne on rounded knobs.

Sterna sparsely setose, cross-impressions weak, without modifications other than a prominent, regularly rounded, setose, tongue-shaped lobe between $\sigma^{7}$ coxae 4 (Fig. 54). Adenostyle on $\sigma^{7}$ femur 1 distinct (Fig. $55)$. Legs long and slender, 1.3-1.4 $\left(\bigcirc^{\top}\right)$ or $0.9-1.0$ 


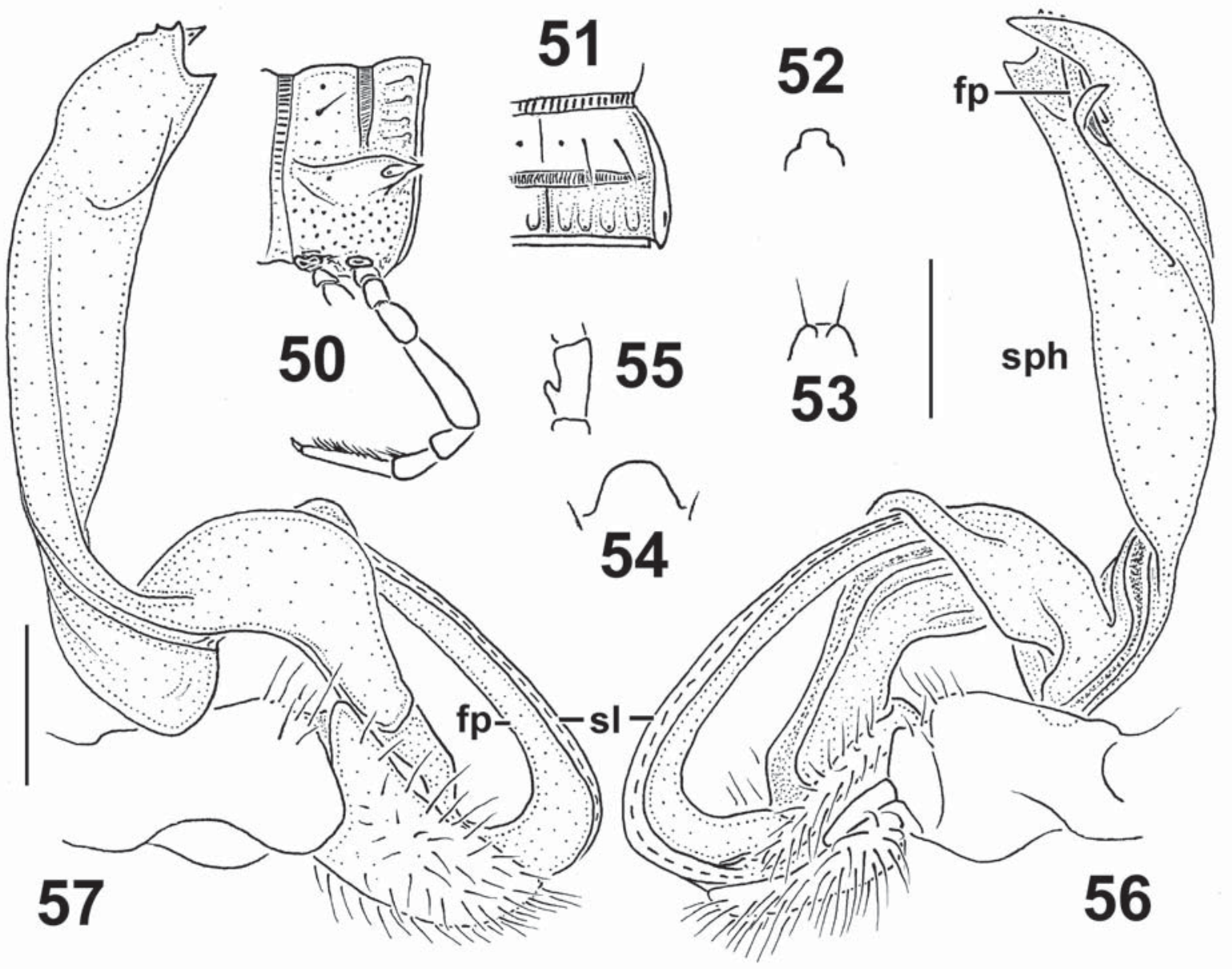

Figs 50-57. Tetracentrosternus martensi sp.n., holotype (50-53) \& $\bigcirc^{7}$ paratype (54-57): 50 — segment 10, lateral view; 51 - right half of segment 10, dorsal view; 52 - tip of epiproct, dorsal view; 53 - hypoproct, ventral view; 54 - sternal lobe between coxae 4, caudal view; 55 - femur 1, lateral view; 56 \& 57 - right gonopod, mesal and lateral views, respectively. Scale bars: 0.5 (50-55) and 0.3 mm (56 \& 57).

Рис. 50-57. Tetracentrosternus martensi sp.n., голотип (50-53) и паратип о (54-57): 50 - сегмент 10, сбоку; 51 — правая половина сегмента 10, сверху; 52 - кончик эпипрокта, сверху; 53 - гипопрокт, снизу; 54 - стернальная пластина между тазиками 4, сзади; 55 - бедро 1, сбоку; 56 и 57 - правый гонопод, соответственно изнутри и сбоку. Масштаб: 0,5 (50-55) и 0,3 мм (56 и 57).

times ( $(+)$ as long as midbody height, $\sigma^{\top}$ prefemora not swollen laterally; in length, femora $>$ tarsi $>$ prefemora $=$ postfemora $=$ tibiae $>$ coxae $($ Figs $45-50)$. Tarsal brushes present only until legs $7\left(\sigma^{7}\right)$.

Gonopods (Figs 56 \& 57) complex, strongly sigmoid, in situ held subparallel to each other; coxite short, about as long as prefemoral (= densely setose) part of telopodite, subcylindrical, setose distally; prefemoral part as usual, densely setose, about $1 / 4$ as long as acropodite; telopodite subgeniculate and clearly twisted in basal half; femorite rudimentary, marking the onset of a femoral process (fp) and a solenomere (sl), both flagelliform, tightly appressed to each other and largely sheathed by a solenophore (sph), apex of fp spiniform and reaching a complex, branched tip of sph.

REMARKS. Likhitrakarn et al. [2013] provided a key to all three heretofore known species of Tetracentrosternus Pocock, 1895. Now it can be somewhat modified to incorporate the above new congener.

1(2) Body smaller: width of midbody metaterga ca $1.0-1.5$ $\mathrm{mm}$. Paraterga poorly-developed, mostly sharp caudally
(Figs 45-51). Gonopod twisted and especially strongly sigmoid, subgeniculate in basal half (Figs 56 \& 57) ..... T. martensi sp.n.

2(1) Body larger: width of midbody metaterga $\geq 2.0 \mathrm{~mm}$. Paraterga either much better developed and then mostly sharp caudally or especially poorly developed and then never sharp caudally. Gonopod neither twisted nor subgeniculate

3(4) Paraterga poorly-developed, not sharp caudally. Metaterga smooth. Sternal cones present. Myanmar

.. T. subspinosus

4(3) Paraterga strongly developed and acute caudally (much like in Figs 50 \& 51). Metaterga rugose or tuberculate at least near caudal margin (much like in Figs 50 \& 51). Sternal cones absent

5(6) $\sigma^{7}$ femur 1 with an adenostyle, however small. Gonopod suberect, with a basal gonofemoral tooth and a distally strongly fimbriate/spiculate solenophore, but without evident processes distal to gonofemoral tooth. Yunnan, southern China

T. hoffmani

6(5) $\sigma^{7}$ femur 1 without an adenostyle. Gonopod strongly elongate and curved distally, supplied with three evident processes distal to femorite. Thailand ...... T. theelorsuensis 


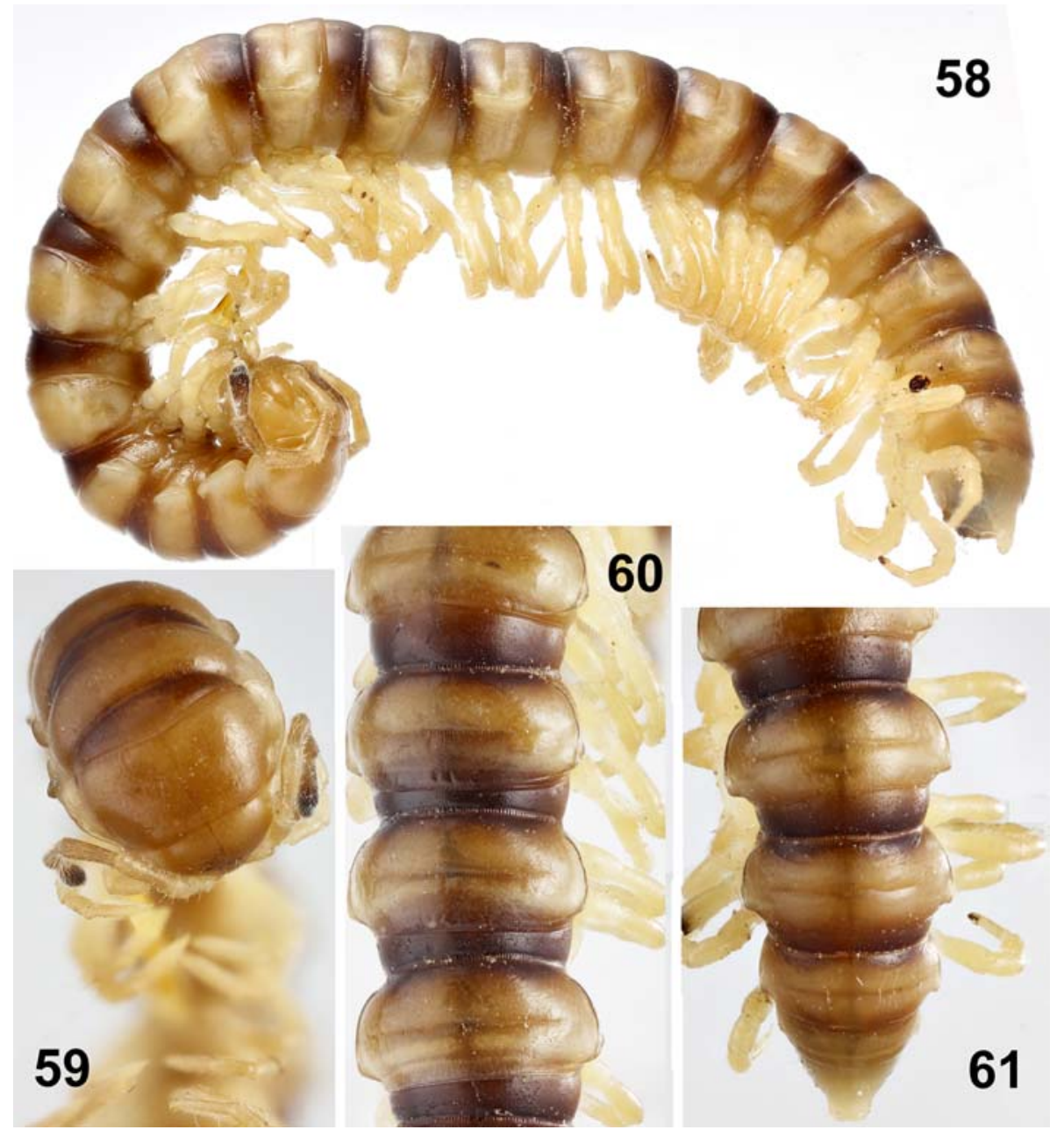

Figs 58-61. Inversispina multispina sp.n., holotype: 58 - habitus, lateral view; 59 - anterior part of body, ventral view; $60-$ middle part of body, dorsal view; 61 - posterior part of body, dorsal view. Pictures by K. Makarov, not taken to scale.

Pис. 58-61. Inversispina multispina sp.n., голотип: 58 - общий вид, сбоку; 59 - передняя часть тела, снизу; 60 - средняя часть тела, сверху; 61 - задняя часть тела, сверху. Фото К. Макарова, сняты без масштаба.

\section{Inversispina multispina sp.n.}

Figs 58-67.

HOLOTYPE O' (ZMUM 03105$)$, China, Sichuan Prov., SSE of Shimian, S of Zhuma, N29 $02^{\prime} 07^{\prime \prime}$, E102 $28^{\prime} 59^{\prime \prime}, 2360$ m a.s.1., 24.V.2014, leg. I. Belousov \& I. Kabak.

DIAGNOSIS. This new species seems to be especially similar to I. trispina Golovatch, 2013, also from Sichuan [Golovatch, 2013a], in sharing such somatic and gonopod characters as the rather poorly-developed paraterga, the long legs and, especially, the presence of a distinct ventral tubercle on the gonopod coxite, as well as of several ventral spines and a dorsal outgrowth at the base of a non-coiled solenophore. Both these species differ readily by a cingulated colour pattern, the even less strongly developed paraterga and, especially, a far more complex solenophore which carries as many as five ventral spines (a-e) and a non-geniculate dorsoparabasal outgrowth (g) in I. multispina sp.n.

NAME. To emphasize the presence of numerous, mostly spiniform processes arising from the solenophore.
DESCRIPTION. Length ca $21 \mathrm{~mm}$, width of midbody pro- and metazonae 2.0 and $2.5 \mathrm{~mm}$, respectively $\left(\mathrm{O}^{7}\right)$. General coloration in alcohol yellowish to brown, with a distinct cingulated pattern of darker brown prozonae and, especially, stricture regions contrasting with yellow to light brown metazonae (Figs 58-61). Antennae brown, increasingly infuscate towards dark brown antennomeres 6 and 7, but tip pallid. Legs, venter and epiproct yellow.

Clypeolabral region sparsely setose, vertigial one bare; epicranial suture thin, superficial (Figs 58 \& 59). Antennae rather long and moderately clavate, in situ slightly extending back behind segment 2 when stretched dorsally $\left(O^{7}\right)$; antennomeres 2-6 subequal in length; interantennal isthmus almost as broad as diameter of antennal socket (Fig. 59).

In width, head $<$ segment $3=4<$ collum $<3<5$ 15; thereafter body gradually tapering towards telson. Tegument smooth and shining, prozonae shagreened, surface below paraterga microgranulate. Collum broadly and regularly rounded laterally. Postcollum paraterga 

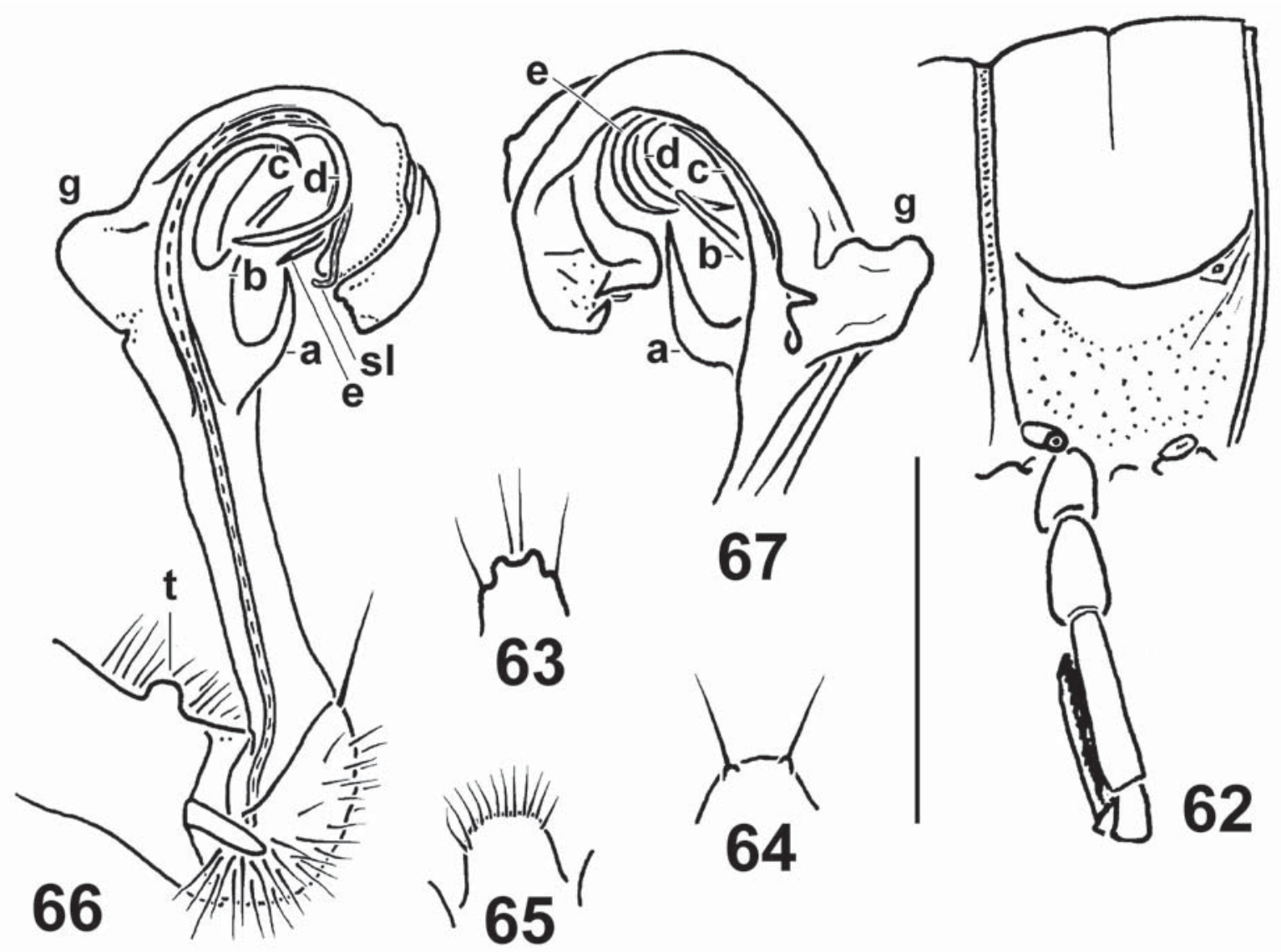

Figs 62-67. Inversispina multispina sp.n., holotype: 62 - segment 10, lateral view; 63 - tip of epiproct, dorsal view; 64 hypoproct, ventral view; 65 - sternal lobe between coxae 4, caudal view; 66 \& 67 - left gonopod, mesal and sublateral views, respectively. Scale bars: $2.0(62-65)$ and $1.0 \mathrm{~mm}(66 \& 67)$.

Рис. 62-67. Inversispina multispina sp.n., голотип: 62 - сегмент 10, сбоку; 63 - кончик эпипрокта, сверху; 64 - гипопрокт, снизу; 65 - стернальная пластина между тазиками 4, сзади; 66 и 67 - левый гонопод, соответственно изнутри и почти сбоку. Масштаб: 2,0 (62-65) и 1,0 мм (66 и 67).

poorly-developed, set low at about upper $1 / 3^{-1 / 2}$ of metazonae, only in segment 2 slightly drawn both anteriorly and posteriorly, following paraterga obtuse-angled and more or less clearly rounded caudally, never drawn behind rear tergal margin; calluses demarcated by a complete distinct sulcus only dorsally, only in about caudal $1 / 3$ by a less distinct sulcus also ventrally; poriferous calluses a little thicker than poreless ones (Figs 58-62). Ozopores lateral, placed inside a round to subtriangular pit near caudal corner. Tergal setae largely abraded, pattern untraceable, only segment 18 with $2+2$ short setae in a transverse fore (= pre-sulcus) row. Transverse metatergal sulci thin, superficial, faintly sigmoid centrally, not reaching bases of paraterga, present on metaterga 5-18 (Figs 58, 60$62)$. Stricture dividing pro- and metazonae thin and deep, faintly striolate at bottom down to paraterga. Pleurosternal carinae and axial line missing. Epiproct (Figs 58, 61 \& 53) rather long, clearly flattened dorsoventrally, conical, concave at apex, subapical lateral papillae evident. Hypoproct (Fig. 64) roundly subtrapeziform, caudal $1+1$ setae well separated, borne on small rounded knobs.
Sterna sparsely setose, cross-impressions weak, without modifications other than a prominent, regularly rounded, setose, tongue-shaped lobe between coxae 4 (Fig. 65). A pair of low rounded bulges anterolateral to gonopod aperture. Legs long and slender, 1.4-1.5 times as long as midbody height $\left(\sigma^{7}\right)$, prefemora not swollen laterally; in length, femora $>$ tarsi $>$ prefemora $=$ postfemora $=$ tibiae $>$ coxae (Fig. 62). Tarsal brushes gradually thinning out towards legs of caudal $1 / 3$ of body. Adenostyles missing.

Gonopods (Figs 66 \& 67) highly complex, in situ held subparallel to each other; coxite short, subcylindrical, ca $1 / 3$ as long as telopodite, setose ventrolaterally, with a distinct tubercle (t) ventrally near midlength; prefemoral (= densely setose) part of telopodite short, shorter than coxite and ca 0.7 times as long as a slender, simple and erect femorite. Distal half of telopodite strongly curved caudad and subcircular, demarcated from femorite by bases of a large, ear-shaped, dorsal outgrowth (g), a free, mesal, flagelliform solenomere (sl) and an unciform ventral process (a); solenophore with a succession of another four (b, c, d and e) ventral, mostly caudally curved, spiniform processes distal to $\mathbf{a}$. 


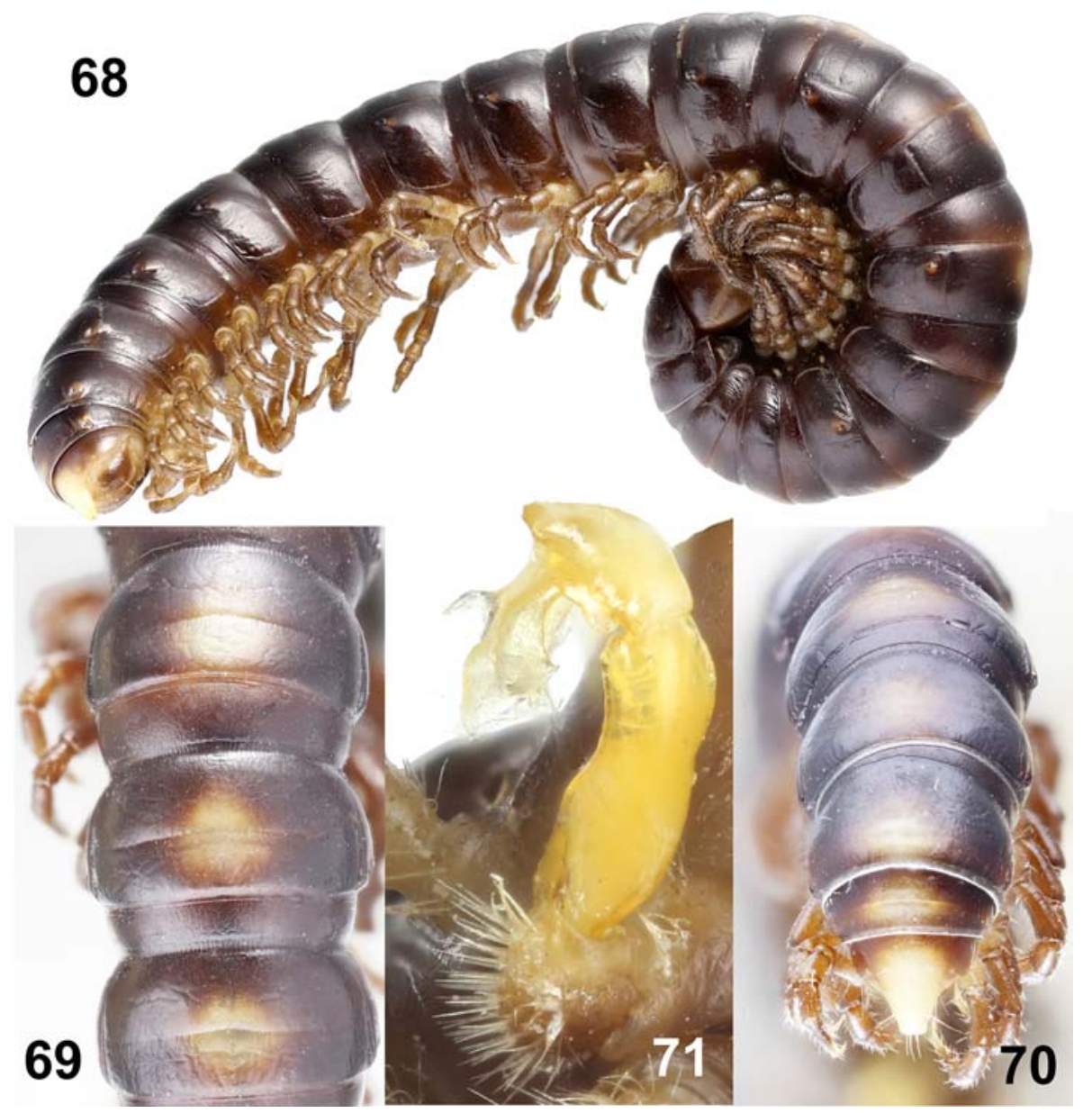

Figs 68-71. Sigipinius pinnifer sp.n., holotype: 68 - habitus, lateral view; 69 - midbody segments, dorsal view; 70 — caudal part of body, dorsal view; 71 - left gonopod in situ, ventrolateral view. Pictures by K. Makarov, not taken to scale.

Рис. 68-71. Sigipinius pinnifer sp.n., голотип: 68 - общий вид, сбоку; 69 - среднетуловищные сегменты, сверху; 70 - задняя часть тела, сверху; 71 - левый гонопод на месте, одновременно снизу и сбоку. Фото К. Макарова, сняты без масштаба.

REMARKS. The genus Inversispina Zhang, in Zhang, Wang et Zhang, 1997, has hitherto been known to contain four species, three of which come from continental China, plus one more from Taiwan [Chen et al., 2011a; Golovatch, 2012, 2013a, b]. The new species fails to alter the presently accepted generic diagnosis [Golovatch, 2012].

\section{Sigipinius pinnifer sp.n.}

Figs 68-78.

HOLOTYPE OT (ZMUM 03107 ), China, Sichuan Prov., SSE of Shimian, S of Zhuma, N29 $0^{\prime} 19^{\prime \prime}$, E102 $27^{\prime} 24^{\prime \prime}, 3625$ m a.s.1., 26.V.2014, leg. I. Belousov \& I. Kabak.

PARATYPE + (ZMUM $\rho 3108)$, same data, together with holotype.

DIAGNOSIS. Differs from all seven hitherto known species of Sigipinius, all from China [see Golovatch, 2013b, 2014a], primarily by the flipper-shaped, mesal, distodorsal outgrowth (f) on a unipartite solenophore (sph).

NAME. To emphasize the flipper-shaped, mesal, distodorsal outgrowth on the solenophore.
DESCRIPTION. Length of holotype ca $21 \mathrm{~mm}$, width of midbody pro- and metazonae 2.4 and $2.7 \mathrm{~mm}$, respectively; length of paratype ca $25 \mathrm{~mm}$, width of midbody pro- and metazonae 2.8 and $3.0 \mathrm{~mm}$, respectively. General coloration in alcohol dark brown to blackish, with a distinct pattern of contrasting yellowish, oblong to roundish, central spots on collum and following metaterga (Figs 68-70). Antennae dark brown to blackish, but tip pallid. Legs and venter brown, epiproct yellow.

Clypeolabral region densely, vertigial one sparsely, setose; epicranial suture deep. Antennae rather short and moderately clavate, in situ slightly extending back behind midlength of segment $2\left(\sigma^{7}\right)$ or behind collum (+) when stretched dorsally; antennomeres 2-6 subequal in length; interantennal isthmus ca 1.2 times as broad as diameter of antennal socket.

In width, collum $<$ segment $3=4<2<$ head $<3<$ $5-16$; thereafter body gradually tapering towards telson. Tegument generally smooth and shining, only in places delicately rugulose, but clearly rugulose to rugose below paraterga; prozonae shagreened. Collum 


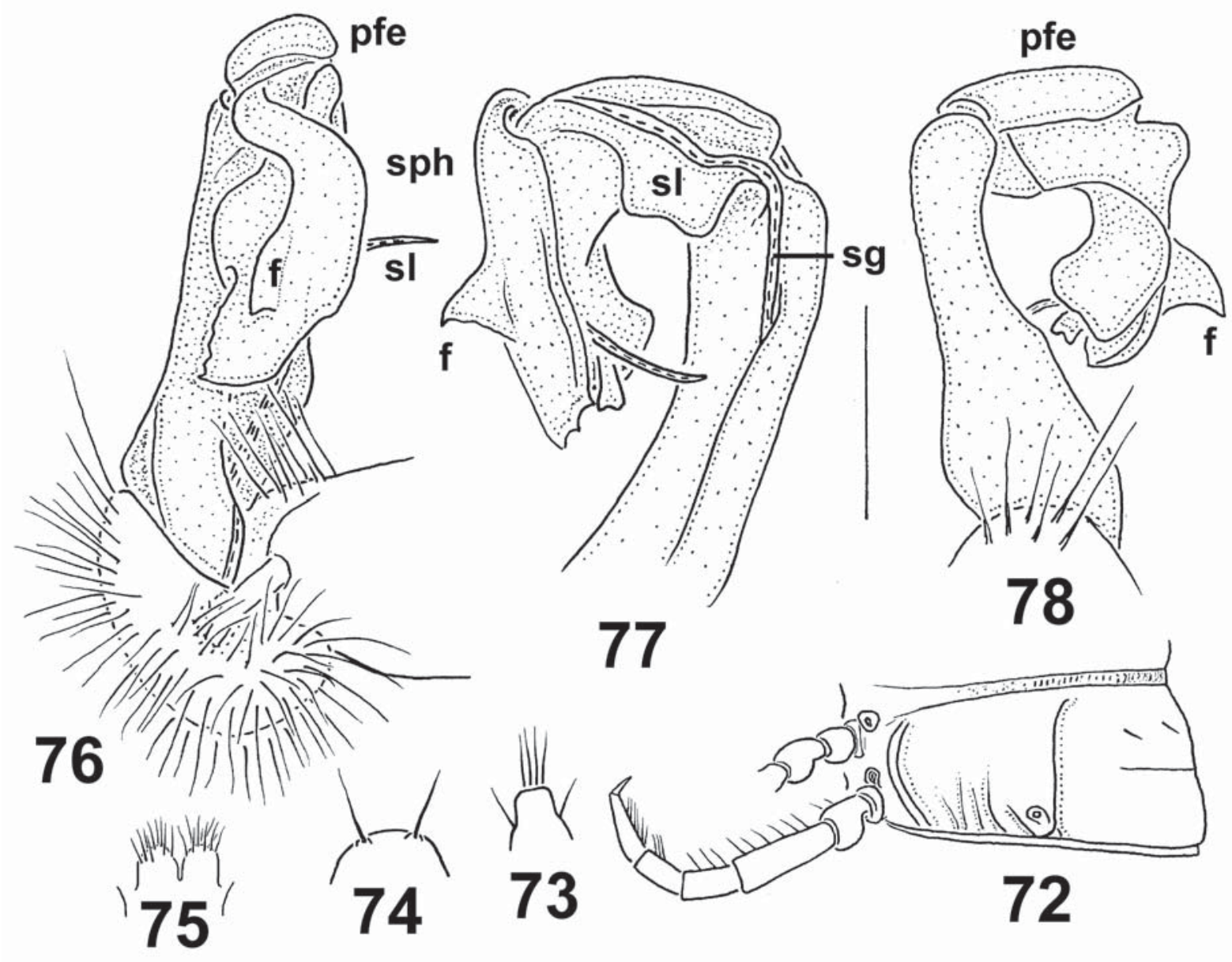

Figs 72-78. Sigipinius pinnifer sp.n., holotype: 72 - segment 10, lateral view; 73 - tip of epiproct, dorsal view; 74 - hypoproct, ventral view; 75 - sternal lobes between coxae 4, caudal view; 76-78 - right gonopod, mesal, dorsal and ventral views, respectively. Scale bars: $1.0(72-75)$ and $0.5 \mathrm{~mm}(76-78)$.

Рис. 72-78. Sigipinius pinnifer sp.n., голотип: 72 - сегмент 10, сбоку; 73 - кончик эпипрокта, сверху; 74 - гипопрокт, снизу; 75 - стернальные пластины между тазиками 4, сзади; 76-78 - правый гонопод, соответственно изнутри, сверху и снизу. Масштаб: 1,0 (72-78) и 0,5 мм (76-78).

broadly and regularly rounded laterally. Postcollum paraterga very poorly-developed, set low at about upper $1 / 3^{-1 / 2}$ of metazonae, only in segment 2 slightly drawn both anteriorly and posteriorly, following paraterga obtuse-angled and more or less clearly rounded caudally, only paraterga 2 particularly thin and drawn both anteriorly and posteriorly, thereafter never produced behind rear tergal margin; calluses demarcated by a complete distinct sulcus only dorsally, only in about caudal $1 / 3^{-1 / 4}$ by a less distinct sulcus also ventrally; calluses typically thick, poriferous calluses a little thicker than poreless ones (Figs 68-72). Ozopores lateral, placed inside an ovoid pit near caudal corner. Tergal setae largely abraded, pattern traceable as $3+3$ short setae in a transverse fore (= pre-sulcus) row; setae ca $1 / 5$ as long as metatergite. Transverse metatergal sulci thin, superficial, not reaching bases of paraterga, present on metaterga 5-18 (Figs 68-70 \& 72). Stricture dividing pro- and metazonae thin and rather shallow, faintly striolate at bottom down to paraterga. Pleurosternal carinae distinct arcuate ridges gradually disappearing only towards segment 19 . Axial line missing. Epiproct (Figs 68, 70 \& 73) rather long, clearly flattened dorsoventrally, conical, subtruncate at apex, subapical lateral papillae small. Hypoproct (Fig. 74) semi-circular, caudal 1+1 setae well separated, borne on very small rounded knobs.

Sterna sparsely setose, cross-impressions weak, without modifications other than two small, densely setose, barely separated, rounded lobes between $\sigma^{7}$ coxae 4 (Fig. 75). Legs short, in $\sigma^{7}$ slightly incrassate, in + slender, 1.2$1.3\left(O^{7}\right)$ or $0.9-1.0$ times $(+)$ as long as midbody height, $\sigma^{7}$ prefemora moderately swollen laterally (Fig. 72); in length, femora $>$ tarsi $>$ prefemora $=$ postfemora $=$ tibiae $>$ coxae (Fig. 72). Tarsal brushes gradually thinning out towards legs of segment 10. Adenostyles missing.

Gonopods (Figs 71 \& 76-78) complex, stout, in situ crossing each other; coxite short, subcylindrical, ca $1 / 3$ as long as telopodite, setose distoventrally; prefemoral (= densely setose) part of telopodite about as long as coxite and half as long as a rather simple and 


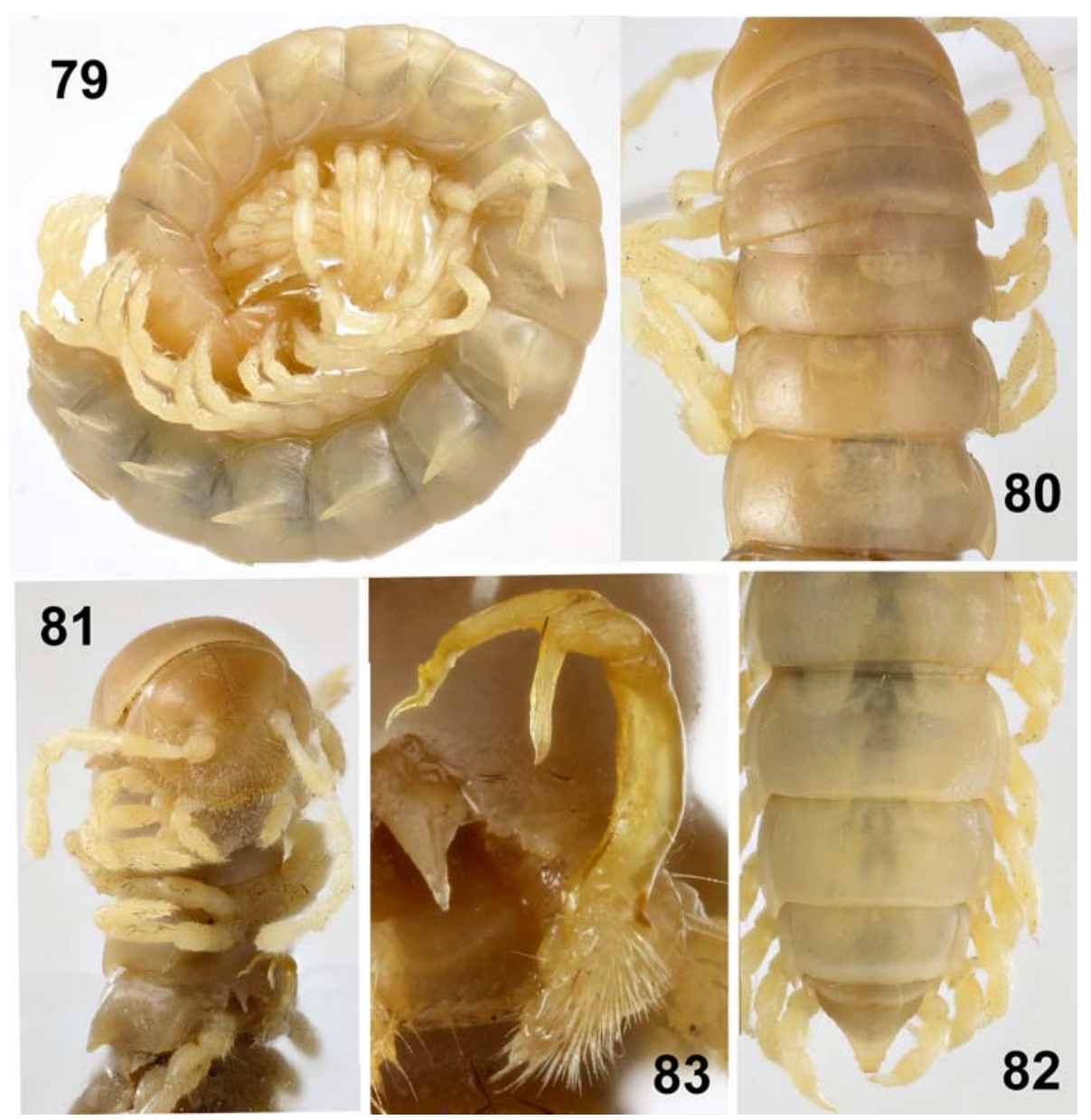

Figs 79-83. Sundanina fedorenkoi sp.n., holotype (79) \& $0^{7}$ paratype (80-83): 79 — habitus, lateral view; $80 \& 81$ — anterior part of body, dorsal and subventral views, respectively; 82 - caudal part of body, dorsal view; 82 - left gonopod in situ, ventrolateral view. Pictures by K. Makarov, not taken to scale.

Рис. 79-83. Sundanina fedorenkoi sp.n., голотип (79) и паратип О7 (80-83): 79 - общий вид, сбоку; 80 и 81 - передняя часть тела, соответственно сверху и одновременно снизу и сбоку; 82 - задняя часть тела, сверху; 83 - левый гонопод на месте, одновременно снизу и сбоку. Фото К. Макарова, сняты без масштаба.

slightly curved femorite. Distal half of telopodite strongly curved mesad, subcircular, demarcated from femorite by a distinct cingulum; a basally enlarged, but mostly flagelliform, free solenomere (sl) and a clearly demarcated postfemoral part (pfe) both starting immediately distal to cingulum, pfe clearly set off apically by a sulcus from a complex lamellar solenophore (sph), the latter broad, unipartite, subacuminate and carrying a characteristic flipper-shaped, mesal, distodorsal outgrowth (f). Seminal groove (sg) running entirely on mesal side of a somewhat hollow and untwisted femorite before passing onto $\mathbf{s l}$.

REMARKS. The new species is a typical member of Sigipinius Hoffman, 1961, and it fails to alter the diagnosis of the genus as reformulated by Golovatch [2013b].

\section{Sundanina fedorenkoi sp.n.}

Figs 79-90.

HOLOTYPE ơ (ZMUM 03109$)$, Vietnam, Dak Lak Prov., Chu Yang Sin National Park, N12 $23^{\prime} 48^{\prime \prime}$, E108 $20^{\prime} 59^{\prime \prime}$, ca 1000 $\mathrm{m}$ a.s.1., 30.III.2014, leg. D. Fedorenko.
PARATYPE $O^{7}$ (ZMUM $\rho 3110$ ), same data, together with holotype.

DIAGNOSIS. Differs from all 14 hitherto known species of Sundanina Attems, 1914, all from Southeast Asia, mainly Sumatra, Indonesia (see Nguyen \& Sierwald, 2013), primarily by the absence of femoral outgrowths on the gonopod, coupled with the presence of a single strong process (= solenophore) at the base of a long, mostly thick and twisted solenomere.

NAME. Honours Dmitri N. Fedorenko, the collector.

DESCRIPTION. Length of holotype ca $22 \mathrm{~mm}$, width of midbody pro- and metazonae 2.5 and $3.0 \mathrm{~mm}$, respectively; length of paratype ca $27 \mathrm{~mm}$, width of midbody pro- and metazonae 3.3 and $4.0 \mathrm{~mm}$, respectively. Coloration in alcohol uniformly light creamy to very light brown (Figs 79-82).

Head densely setose nearly throughout (Fig. 81); epicranial suture evident. Antennae long and slender, only moderately clavate, in situ extending back behind segment $3\left(O^{7}\right)$ when stretched dorsally; antennomeres 


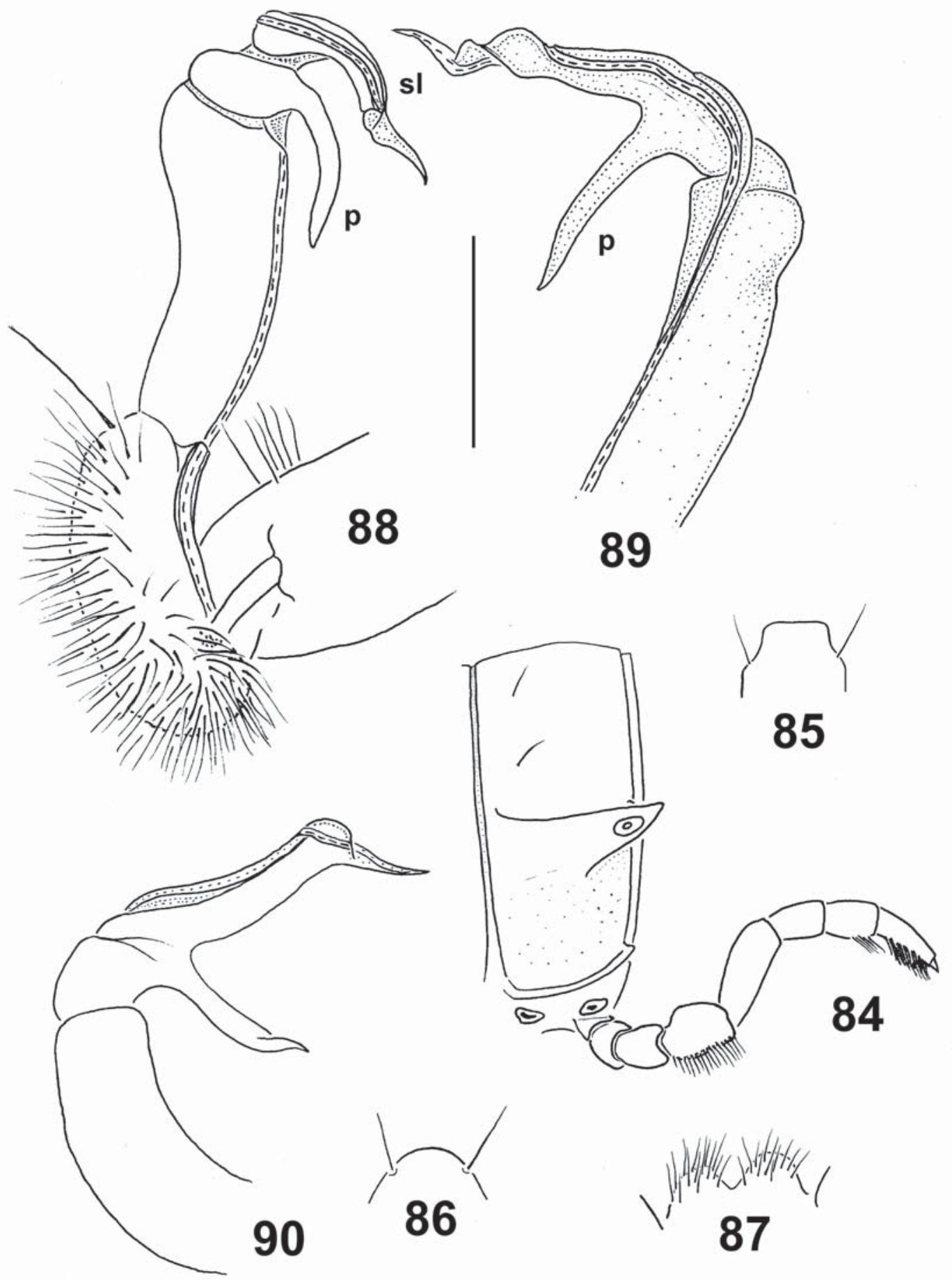

Figs 84-90. Sundanina fedorenkoi sp.n., $\mathrm{O}^{7}$ paratype: 84 - segment 10, lateral view; 85 - tip of epiproct, dorsal view; 86 hypoproct, ventral view; 87 - sternal lobes between coxae 4, caudal view; 88-90 - right gonopod, mesal, dorsal and ventral views, respectively. Scale bars: $1.0(84-87)$ and $0.5 \mathrm{~mm}(88-90)$.

Рис. 84-90. Sundanina fedorenkoi sp.n., паратип О7 84 - сегмент 10, сбоку; 85 - кончик эпипрокта, сверху; 86 - гипопрокт, снизу; 87 - стернальные пластины между тазиками 4, сзади; 88-90 - правый гонопод, соответственно изнутри, сверху и снизу. Масштаб: 1,0 (84-87) и 0,5 мм (88-90).

2-6 subequal in length; interantennal isthmus ca 1.5 times as broad as diameter of antennal socket (Fig. 81).

In width, head $=$ collum $=$ segment $3=4<2<5-$ 16; thereafter body gradually tapering towards telson.
Tegument generally smooth and shining, only below paraterga microgranulate; prozonae shagreened. Collum with caudal corner of paraterga acute-angled and narrowly rounded. Postcollum paraterga strongly de- 
veloped, set low at about upper $1 / 3^{-1 / 2}$ of metazonae, only in segment 2 drawn not only posteriorly, but also anteriorly, caudal corners invariably acute, sharp and produced well beyond rear tergal margins; lateral calluses subqually thin on poriferous and poreless paraterga, demarcated by a complete distinct sulcus only dorsally, only in about caudal $1 / 3$ by a less distinct sulcus also ventrally (Figs 79-82 \& 84). Ozopores lateral, placed inside an ovoid pit near caudal corner. Tergal setae largely abraded, pattern traceable as $2+2$ short setae or insertion points in a transverse fore row on metaterga; setae ca $1 / 4$ as long as metatergite. Transverse metatergal sulci absent (Figs 79-82 \& 84). Stricture dividing pro- and metazonae thin and shallow, smooth to very faintly striolate at bottom down to paraterga. Pleurosternal carinae distinct arcuate ridges with a small caudal tooth on segments $2-18$, tooth absent only from segment 19. Axial line missing. Epiproct (Figs 79, $82 \& 85$ ) rather long, clearly flattened dorsoventrally, conical, subtruncate at apex, subapical lateral papillae small. Hypoproct (Fig. 86) semicircular, caudal $1+1$ setae well separated, not borne on knobs.

Sterna very densely setose, cross-impressions weak, near each coxa with a minute cone, the latter a little more distinct near each caudal coxa; two small, densely setose, medially subcontiguous, rounded lobes between coxae 4 (Fig. 87). Legs long, in $\sigma^{7}$ apparently slightly incrassate, 1.4-1.5 times as long as midbody height $\left(\sigma^{7}\right)$,prefemora swollen laterally $\left(\sigma^{7}\right)$ (Fig. 84); in length, femora $>$ tarsi $>$ prefemora $=$ postfemora $=$ tibiae $>$ coxae. All podomeres densely setose ventrally, forming brushes on prefemora, tibiae and tarsi. Adenostyles missing.

Gonopods (Figs 83 \& 88-90) simple, in situ crossing each other; coxite medium-sized, subcylindrical, ca $1 / 3$ as long as telopodite, setose distoventrally; prefemoral (= densely setose) part of telopodite about as long as coxite and half as long as a simple, slender and only slightly curved femorite. Distal half of telopodite strongly curved mesad, demarcated from femorite by a distinct cingulum; acropodite represented by a long parabasal process (p) (= solenophore) and a long, thick, gradually attenuated, distally twisted and apically acuminate solenomere (sl).

REMARKS. This new species seems to be especially similar to $S$. medialis Nguyen, 2010 and $S$. cochinchina Nguyen, 2010, both also from Vietnam and both showing a large process arising from the base of the solenophore [Nguyen, 2010]. However, unlike these two species, $S$. fedorenkoi sp.n. is totally devoid of outgrowths arising immediately basal to the apical demarcation cingulum/sulcus of the gonofemorite, whereas the solenophore is only represented by a large parabasal process (p) and the solenomere (sl) is not so much flagelliform, but thick (more like in S. cochinchi$n a$ ), long and clearly twisted distally. It is also noteworthy that, based on gonopod conformation alone, $S$. fedorenkoi sp.n. must be regarded as a quite disjunct member not only of the genus Sundanina, but also of the tribe Sundaninini it belongs in.

Surprisingly, the long, distally twisted, thick and gradually attenuating solenomere in S. fedorenkoi sp.n. strongly resembles the one observed in the monobasic genus Simplogonomorpha Nguyen et Korsós, 2011, with $S$. falcata (Attems, 1953), also from Vietnam [Nguyen, Korsós, 2011], but too many other characters, both somatic and gonopodal, are so disparate that Simplogonomorpha is assigned to the tribe Chamberliniini.

ACKNOWLEDGEMENTS. Special thanks go to Louis Deharveng and Anne Bedos (both MNHN), Igor Belousov and I. Kabak (both St. Petersburg, Russia), Leonardo Latella (MCSNV), Jochen Martens (Mainz, Germany), Peter Jäger (SMF), Igor Muratov (Pietermaritzburg, South Africa), Igor Melnik (Moscow, Russia) and Dmitri Fedorenko (Institute for Problems of Ecology and Evolution, Russian Academy of Sciences, Moscow, Russia) for the provision of material treated above. Kirill Makarov (Moscow, Russia) very skillfully took most of the pictures while Didier VandenSpiegel and Jonathan Brecko (both MRAC) arranged the pictures of Chamberlinius vietnamicus sp.n. to be taken. My cordial thanks go to Kirill Mikhailov and Elena Kudryavtseva (both ZMUM) who helped me incorporate the ZMUM samples into the collection. Dmitri Fedorenko extends his gratitude to the Russia-Vietnam Joint Tropical Centre, which supported his collecting trips to Vietnam. Louis Deharveng and Anne Bedos are grateful to Pham Dinh Sac (Institute of Ecology and Biological Resources, Hanoi, Vietnam) who organized the 2011 field trip for "a survey of cave invertebrate fauna in the Phong Nha - Ke Bang National Park region" as part of a larger project, "Nature Conservation and Sustainable Natural Resource Management in the Phong Nha - Ke Bang Region, Vietnam, KfW component", funded by the German development bank KfW.

\section{References}

Attems C. 1937. Myriopoda 3. Polydesmoidea I. Fam. Strongylosomidae // Das Tierreich. Lfg.68. S.I-XXII, 1-300.

Attems C. 1938. Die von Dr. C. Dawydoff in Französisch Indochina gesammelten Myriopoden // Mémoires du Muséum national d'Histoire naturelle, N.S. T.6. Fasc.2. P.187-353.

Chen C.C., Golovatch S.I., Chang H.W. 2011a. The genus Inversispina Zhang, in Zhang, Wang et Zhang, 1997, new to the millipede fauna of Taiwan, with the description of a new species (Diplopoda: Polydesmida: Paradoxosomatidae: A1ogolykinae) // Arthropoda Selecta. Vol.20. No.4. P.251-257.

Chen C.C., Golovatch S.I., Chang H.W., Chen S.H. 2011b. Revision of the Taiwanese millipede genus Chamberlinius Wang, 1956, with descriptions of two new species and a reclassification of the tribe Chamberlinini (Diplopoda, Polydesmida, Paradoxosomatidae, Paradoxosomatinae) // ZooKeys. Vol.98. P.1-27.

Golovatch S.I. 1994. Diplopoda from the Himalayas. Two new Alogolykini (Polydesmida: Paradoxosomatidae) // Senckenbergiana biologica. Bd.73. H.1-2. S.183-187.

Golovatch S.I. 2009a. On several new or poorly-known Oriental Paradoxosomatidae (Diplopoda: Polydesmida), VIII // Arthropoda Selecta. Vol.18. Nos 1-2. P.1-7.

Golovatch S.I. 2009b. On several new or poorly-known Oriental Paradoxosomatidae (Diplopoda: Polydesmida), IX // Arthropoda Selecta. Vol.18. Nos 3-4. P.119-124. 
Golovatch S.I. 2012. On several new or poorly-known Oriental Paradoxosomatidae (Diplopoda: Polydesmida), XII // Arthropoda Selecta. Vol.21. No.1. P.1-12.

Golovatch S.I. 2013a. On several new or poorly-known Oriental Paradoxosomatidae (Diplopoda: Polydesmida), XIII // Arthropoda Selecta. Vol.22. No.1. P.1-31.

Golovatch S.I. 2013b. On several new or poorly-known Oriental Paradoxosomatidae (Diplopoda: Polydesmida), XIV // Arthropoda Selecta. Vol.22. No.4. P.307-332.

Golovatch S.I. 2014a. On several new or poorly-known Oriental Paradoxosomatidae (Diplopoda: Polydesmida), XV // Arthropoda Selecta. Vol.23. No.1. P.1-19.

Golovatch S.I. 2014b. On several new or poorly-known Oriental Paradoxosomatidae (Diplopoda: Polydesmida), XVI // Arthropoda Selecta. Vol.23. No.3. P.227-251.

Golovatch S.I. 2015. On several new or poorly-known Oriental Paradoxosomatidae (Diplopoda: Polydesmida), XVII // Arthropoda Selecta. Vol.24. No.2. P.127-168.

Golovatch S.I. 2016. On several new or poorly-known Oriental Paradoxosomatidae (Diplopoda: Polydesmida), XVIII // Arthropoda Selecta. Vol.25. No.1. P.1-18.

Golovatch S.I., Semenyuk I.I. 2010. On several new or poorlyknown Oriental Paradoxosomatidae (Diplopoda: Polydesmida), X // Arthropoda Selecta. Vol.19. No.3. P.123-127.

Golovatch S.I., Wesener T. 2016 (in press). A species checklist of the millipedes (Diplopoda) of India // Zootaxa.

Hoffman R.L. 1963. A contribution to the knowledge of Asiatic strongylosomoid Diplopoda (Polydesmida: Strongylosomatidae) // Annals and Magazine of Natural History. Ser.13. Vol.5. P.577-593.

Jeekel C.A.W. 1965. A revision of the Burmese Paradoxosomatidae (Diplopoda, Polydesmida) in the Museo Civico di Stotia Naturale at Genoa (Part 1) // Tijdschrift voor Entomologie. Deel 108. Afl.2. P.95-144.

Likhitrakarn N., Golovatch S.I., Panha S. 2013. The millipede genus Tetracentrosternus Pocock, 1895 (Polydesmida, Para- doxosomatidae, Alogolykinae, Alogolykini), with a description of the first, new species from Thailand// ZooKeys. Vol.358. P.1-10.

Likhitrakarn N., Golovatch S.I., Panha S. 2015. Review of the millipede genus Kronopolites Attems, 1914 (Diplopoda, Polydesmida, Paradoxosomatidae), with the description of a new species from Laos // ZooKeys. Vol.472. P.27-41.

Likhitrakarn N., Golovatch S.I., Panha S. 2016. Review of the Southeast Asian millipede genus Antheromorpha Jeekel, 1968 (Diplopoda, Polydesmida, Paradoxosomatidae) // ZooKeys. Vol.571. P.21-57.

Nakamura Y., Korsós Z. 2010. Distribution and diversity of millipedes of the Rykyu Archipelago, with the Senkaku and Daito island groups: A literature review (Arthropoda: Diplopoda) // Acta Arachnologica. Vol.59. No.2. P.73-86.

Nguyen D.A. 2010. The millipede tribe Sundaninini in Vietnam (Diplopoda: Polydesmida: Paradoxosomatidae) // Zootaxa. Vol.2479. P.59-68.

Nguyen D.A., Korsós Z. 2011. A revision of the millipede genus Riukiupeltis Verhoeff, 1939 (Diplopoda, Polydesmida, Paradoxosomatidae), with comments on the status of related species // ZooKeys. Vol.156. P.25-40.

Nguyen D.A., Sierwald P. 2013. A worldwide catalog of the family Paradoxosomatidae Daday, 1889 (Diplopoda: Polydesmida) // Check List. Vol.9. No.6. P.1132-1353.

Murakami Y. 1975. The cave myriapods from the Ryukyu Islands (I) // Bulletin of the National Science Museum, Ser. A (Zoology). Vol.1. No.2. P.85-113.

Pocock R.I. 1895. The Myriapoda of Burma, Pt. 4. Report upon the Polydesmoidea collected by Sig. L. Fea, Mr. E. W. Oates and others // Annali del Museo Civico di Storia Naturale di Genova. T.34. P.787-834.

Responsible editor K.G. Mikhailov 